\title{
CENTRAL CHARGES OF T-DUAL BRANES FOR TORIC VARIETIES
}

\author{
BOHAN FANG
}

\begin{abstract}
Given any equivariant coherent sheaf $\mathcal{L}$ on a compact semi-positive toric orbifold $\mathcal{X}$, its SYZ T-dual mirror dual is a Lagrangian brane in the Landau-Ginzburg mirror. We prove the oscillatory integral of the equivariant superpotential in the Landau Ginzburg mirror over this Lagrangian brane is the genus-zero 1-descendant Gromov-Witten potential with a Gamma-type class of $\mathcal{L}$ inserted.
\end{abstract}

\section{INTRODUCTION}

Mirror symmetry relates Gromov-Witten invariants to more classical objects such as period integrals. For an $n$-dimensional toric orbifold $\mathcal{X}$, its mirror is a Landau-Ginzburg model $W:\left(\mathbb{C}^{*}\right)^{n} \rightarrow \mathbb{C}$, where the superpotential $W$ is a Laurent polynomial.

There are many ways to extract Gromov-Witten invariants from this B-model, like quantum cohomology [13, 14, 39] and genus-zero descendant invariants 6, 7 , 17 19, 31 33. In this paper, we would be particularly interested in what the following integration says about the A-model Gromov-Witten theory:

$$
\int_{\Xi} e^{-\frac{W}{z}} \Omega, \quad \Omega=\frac{d X_{1}}{X_{1}} \ldots \frac{d X_{n}}{X_{n}} .
$$

1.1. Motivation. This paper is motivated from several aspects.

1.1.1. SYZ T-duality and homological mirror symmetry. Kontsevich's homological mirror symmetry [26, 27] relates the bounded derived category of coherent sheaves on a complete toric variety (or orbifold) $\mathcal{X}$ to the derived Fukaya-Seidel type category of its mirror Landau-Ginzburg model. There is a correspondence

$$
\operatorname{DFS}\left(\left(\mathbb{C}^{*}\right)^{n}, W\right) \cong D^{b} \operatorname{Coh}(\mathcal{X}) .
$$

Here FS is a kind of Fukaya-Seidel category, and Coh is the category of coherent sheaves. This statement is proved using different setups of Fukaya-Seidel categories 1,10.

There are more structures in these categories of branes. There should be a $B P S$ central charge or simply central charge associated to each brane, either in A or B-model. These central charges play important roles in the stability conditions of Bridgeland [4.

The canonical central charge of a Lagrangian submanifold $\Xi$ in a Calabi-Yau manifold (an A-brane) is the integration of the Calabi-Yau form

$$
\int_{\Xi} \Omega \text {. }
$$


The choice of the central charge reflects the complex structure of the Calabi-Yau manifold, manifested by the Calabi-Yau form here. On the mirror side the central charge of a B-model brane a.k.a. a coherent sheaf is part of the stability condition which in principle should specify the stringy Kähler structure. The definition should involve the quantum correction by Gromov-Witten invariants. In 20, Hosono prescribes the central charges formula for certain Calabi-Yaus. The formula for a B-brane involves a hypergeometric series which comes from Gamma classes [22,25] and mirror symmetry's $I$-function. These central charges for the A and B-branes should be equal under mirror symmetry.

In this paper we will look at the central charges for the mirror pair of a complete semi-positive toric orbifold and its mirror Landau-Ginzburg model. We express the central charge of a brane in $D^{b} \operatorname{Coh}(\mathcal{X})$ as a genus-zero descendant Gromov-Witten potential as in 22, and prove it is equal to the integral (11) on the mirror Lagrangian brane in the Landau-Ginzburg model (central charge on the Lagrangian brane).

1.1.2. Integral structures. Iritani extensively investigates the oscillatory integral (11) in [22]. The integral cycles $\Xi \in H_{n}(X, \operatorname{Re}(W) \gg 0 ; \mathbb{Z})$ form a B-model integral structure. The genus-zero Gromov-Witten defines the quantum cohomology $\left(H_{\mathrm{CR}}^{*}(\mathcal{X}), \circ_{\tau}\right)$. The quantum D-module is a flat connection on the trival bundle $H_{\mathrm{CR}}^{*}(\mathcal{X}) \times H_{\mathrm{CR}}^{*}(\mathcal{X}) \rightarrow H_{\mathrm{CR}}^{*}(\mathcal{X})$ with a parameter $z \in \mathbb{C}^{*}$, called the Dubrovin connection

$$
\nabla_{\alpha}=\partial_{\alpha}+\frac{1}{z} \alpha \circ_{\tau}, \quad \alpha \in H_{\mathrm{CR}}^{*}(\mathcal{X}) .
$$

This connection can be extended to a flat connection on the trivial $H_{\mathrm{CR}}^{*}(\mathcal{X})$-bundle on $H_{\mathrm{CR}}^{*}(\mathcal{X}) \times \mathbb{C}^{*}$. For any $V \in K(\mathcal{X})$, one associates a flat section $\mathcal{Z}(V)(\tau, z)$. All such $\mathcal{Z}(V)$ define an integral lattice in the space of flat sections of $\nabla$. The Bmodel D-module and its integral structure is naturally generated by the lattice $H_{n}\left(\mathcal{Y}_{q}, \operatorname{Re}(W / z) \gg 0 ; \mathbb{Z}\right)$, where $\mathcal{Y}_{q} \cong\left(\mathbb{C}^{*}\right)^{\operatorname{dim} \mathcal{X}}$ is the mirror Landau-Ginzburg model of $\mathcal{X}$, parametrized by the complex parameter $q$. The identification of the D-modules also identifies such integral structures [22].

As noted in [22, the correspondence should come from homological mirror symmetry. Indeed, Iritani proves the identification of the integral structures by first studying the case when $V$ is either $\mathcal{O}_{\mathcal{X}}$ or the skyscraper sheaf. In either case, the corresponding cycle $\Xi$ in Equation (1) is the Strominger-Yau-Zaslow dual to $V$ [2, 30, 37, i.e. cotangent fiber (viewing $\left.\mathcal{Y}_{q} \cong\left(\mathbb{C}^{*}\right)^{n}=T^{*}\left(S^{1}\right)^{n}\right)$ or the base $\left(S^{1}\right)^{n} \subset \mathcal{Y}_{q}$. This identification follows the theme of homological mirror symmetry for toric varieties [1,911. Let $Z(V)$ be the integration of $\mathcal{Z}(V)$ over $\mathcal{X}$ (pairing with $\mathcal{X}$ 's fundamental class). The value of $Z(V)$ is directly computed as a descendant potential

$$
Z(V)=\left\langle\left\langle\frac{\boldsymbol{\Gamma}_{z}(V)}{z(z+\psi)}\right\rangle_{0,1}^{\mathcal{X}},\right.
$$

where $\boldsymbol{\Gamma}_{z}$ is a transcendental characteristic class involving the Gamma function. On the B-side, for example when $V$ is the structure sheaf $\mathcal{O}_{\mathcal{X}}$, Iritani obtains the following under the mirror map when $\tau \in H_{\mathrm{CR}}^{\leq 2}(\mathcal{X} ; \mathbb{C})$

$$
Z\left(\mathcal{O}_{\mathcal{X}}\right)=\int_{\Xi\left(O_{\mathcal{X}}\right)} e^{-W / z} \Omega .
$$

Here SYZ dual $\Xi\left(\mathcal{O}_{\mathcal{X}}\right)$ is $\left(\mathbb{R}_{>0}\right)^{n} \subset \mathcal{Y}_{q} \cong\left(\mathbb{C}^{*}\right)^{n}$. Homological mirror symmetry says $\Xi\left(\mathcal{O}_{\mathcal{X}}\right)$ is the Lagrangian brane in the Landau-Ginzburg model mirror to the coherent sheaf $\mathcal{O}_{\mathcal{X}}$ in $D^{b} \operatorname{Coh}(\mathcal{X})$. Moreover, Iritani also matches Galois actions on 
A and B-sides to generate and match all integral structures, i.e. two "tensoring a line bundle" operations on both sides.

In this paper, we develop this correspondence of central charges more carefully. We show an equivariant version of the above correspondence with an explicit description of $\Xi(\mathcal{L})$ for any $\mathcal{L} \in D^{b} \operatorname{Coh}_{\mathbb{T}}(\mathcal{X})$, the derived category of equivariant coherent sheaves on $\mathcal{X}$. 11 The explicit description of $\Xi(\mathcal{L})$ is the same as the Lagrangian brane obtained in the theorem of homological mirror symmetry of 10 .

\subsection{Main theorem.}

Theorem. Let $\mathcal{X}$ be a proper semi-positive toric orbifold, $\widetilde{W}$ be the equivariantly perturbed superpotential, which is a holomorphic function on the universal cover $\widetilde{\mathcal{Y}}_{q}$ of $\mathcal{Y}_{q}$. Under the mirror map $\boldsymbol{\tau}=\boldsymbol{\tau}\left(q_{0}, q\right)$ (as in [17, 19, 31, 33])

$$
\left\langle\left\langle\frac{\kappa(\mathcal{L})}{z(z+\psi)}\right\rangle_{0,1}^{\mathcal{X}}=\int_{\Xi(\mathcal{L})} e^{-\frac{\widetilde{W}}{z}} \Omega .\right.
$$

The symbol $\left\langle\langle\rangle_{0,1}^{\mathcal{X}}\right.$ is the notation of genus-zero primary and descendant GromovWitten potential with 1 marked point (see Definition 5.1). Here $\Xi(\mathcal{L})$ is a piecewise linear conical Lagrangian cycle in $\widetilde{\mathcal{Y}}_{q}$, and is the conical limit of the equivariant $S Y Z T$-dual brane of $\mathcal{L} 2$

Remark 1.1. The class $\kappa(\mathcal{L})$ related to an equivariant version of the Gamma class (12).

Remark 1.2. This theorem should have some implications, at least in principle, on the Gamma II conjecture proposed by Galkin-Golyshev-Iritani [15] for semi-positive complete toric orbifolds. A proof of the K-theoretic version is in [16, p19]. Such conjecture states when the non-equivariant quantum cohomology is semisimple, like in the complete toric case, the fundamental solutions to the QDEs have certain asymptotic behavior prescribed by the non-equivariant limit of $\kappa$-classes of coherent sheaves in an exceptional collection of $D^{b} \operatorname{Coh}(\mathcal{X})$. The existence of the exceptional collection in the toric case is known [24, while recent works [29, 41] allow us to take non-equivariant limit for coherent-constructible correspondences (see also 28, 36, [40]).

Remark 1.3. One can simply integrate over the SYZ T-dual brane SYZ $(\mathcal{L})$ instead of $\Xi(\mathcal{L})$ and get the same result since it is homotopic to and has the same asymptotic behavior as $\Xi(\mathcal{L})$ (see Remark 3.8).

Remark 1.4. In [12, Liu, Zong and the author directly compute the oscillatory integrals when $\mathcal{X}$ is $\mathbb{P}^{1}$. Combining with the Eynard-Orantin recursion, one can obtain all genus descendant potentials of $\mathbb{P}^{1}$ by integrating the Eynard-Orantin higher genus invariants over the suitable cycles.

1.3. Outline. We fix the notion of compact semi-positive toric orbifolds in Section 2, and recall some basic facts of homological mirror symmetry for complete toric orbifolds in Section 3. In Section 4 we compute the oscillatory integrals by fixing the coefficients due to the fact that the integral is a solution to the GKZ system. We prove the main theorem in Section 5 by invoking the genus-zero mirror theorem of Givental and Lian-Liu-Yau [17, 19, 31, 33.

\footnotetext{
${ }^{1}$ For the cycle mirror to the structure sheaf, Iritani computes $Z\left(\mathcal{O}_{\mathcal{X}}\right)$ equivariantly first and then takes non-equivariant limit.

${ }^{2} \mathrm{~A}$ conical set is a dilation-invariant set in certain direction. See Definition 3.1
} 
1.4. Acknowledgement. The author would like to thank Chiu-Chu Melissa Liu and Zhengyu Zong for valuable discussion. The author would also like to thank Hiroshi Iritani and Eric Zaslow for very helpful comments. This work is partially supported by the Recruitment Program of Global Experts in China and a start-up grant at Peking University.

\section{Smooth Toric DM Stacks}

In this section, we follow the definitions in [22, Section 3.1], with slightly different notation. We work over $\mathbb{C}$.

2.1. Definition. Let $N \cong \mathbb{Z}^{n}$ be a finitely generated free abelian group, and let $N_{\mathbb{R}}=N \otimes_{\mathbb{Z}} \mathbb{R}$. We consider complete toric orbifolds which have trivial generic stabilizers. A toric orbifold is given by the following data:

- vectors $b_{1}, \ldots, b_{r^{\prime}} \in N$. We require the subgroup $\oplus_{i=1}^{r^{\prime}} \mathbb{Z} b_{i}$ is of finite index in $N$.

- a complete simplicial fan $\Sigma$ in $N_{\mathbb{R}}$ such that the set of 1-cones is

$$
\left\{\rho_{1}, \ldots, \rho_{r^{\prime}}\right\}
$$

where $\rho_{i}=\mathbb{R}_{\geq 0} b_{i}, i=1, \ldots, r^{\prime}$.

The datum $\boldsymbol{\Sigma}=\left(\Sigma,\left(b_{1}, \ldots, b_{r^{\prime}}\right)\right)$ is the stacky fan $[3]$. We choose additional vectors $b_{r^{\prime}+1}, \ldots, b_{r}$ such that $b_{1}, \ldots, b_{r}$ generate $N$. There is a surjective group homomorphism

$$
\begin{aligned}
& \phi: \quad \widetilde{N}:=\oplus_{i=1}^{r} \mathbb{Z} \widetilde{b}_{i} \longrightarrow N, \\
& \widetilde{b}_{i} \quad \mapsto b_{i} .
\end{aligned}
$$

Define $\mathbb{L}:=\operatorname{Ker}(\phi) \cong \mathbb{Z}^{k}$, where $k:=r-n$. Then we have the following short exact sequence of finitely generated abelian groups:

$$
0 \rightarrow \mathbb{L} \stackrel{\psi}{\longrightarrow} \widetilde{N} \stackrel{\phi}{\longrightarrow} N \rightarrow 0 .
$$

Applying $-\otimes_{\mathbb{Z}} \mathbb{C}^{*}$ and $\operatorname{Hom}(-, \mathbb{Z})$ to $(2)$, we obtain two exact sequences of abelian groups:

$$
\begin{aligned}
& 1 \rightarrow G \rightarrow \widetilde{\mathbb{T}} \rightarrow \mathbb{T} \rightarrow 1, \\
& 0 \rightarrow M \stackrel{\phi^{\vee}}{\rightarrow} \widetilde{M} \stackrel{\psi^{\vee}}{\rightarrow} \mathbb{L}^{\vee} \rightarrow 0,
\end{aligned}
$$

where

$$
\mathbb{T}=N \otimes_{\mathbb{Z}} \mathbb{C}^{*}=N \otimes_{\mathbb{Z}} \mathbb{C}^{*} \cong\left(\mathbb{C}^{*}\right)^{n}, \widetilde{\mathbb{T}}=\widetilde{N} \otimes_{\mathbb{Z}} \mathbb{C}^{*} \cong\left(\mathbb{C}^{*}\right)^{r}, G=\mathbb{L} \otimes_{\mathbb{Z}} \mathbb{C}^{*} \cong\left(\mathbb{C}^{*}\right)^{k},
$$$$
M=\operatorname{Hom}(N, \mathbb{Z})=\operatorname{Hom}\left(\mathbb{T}, \mathbb{C}^{*}\right), \widetilde{M}=\operatorname{Hom}(\widetilde{N}, \mathbb{Z})=\operatorname{Hom}\left(\widetilde{T}, \mathbb{C}^{*}\right), \mathbb{L}^{\vee}=\operatorname{Hom}(\mathbb{L}, \mathbb{Z})=\operatorname{Hom}\left(G, \mathbb{C}^{*}\right) .
$$

The action of $\widetilde{\mathbb{T}}$ on itself extends to a $\widetilde{\mathbb{T}}$-action on $\mathbb{C}^{r}=\operatorname{Spec} \mathbb{C}\left[Z_{1}, \ldots, Z_{r}\right]$. The group $G$ acts on $\mathbb{C}^{r}$ via the group homomorphism $G \rightarrow \widetilde{\mathbb{T}}$ in (3).

Define the set of "anti-cones"

$$
\mathcal{A}=\left\{I \subset\{1, \ldots, r\}: \sum_{i \notin I} \mathbb{R}_{\geq 0} b_{i} \text { is a cone of } \Sigma\right\} .
$$

Given $I \in \mathcal{A}$, let $\mathbb{C}^{I}$ be the subvariety of $\mathbb{C}^{r}$ defined by the ideal in $\mathbb{C}\left[Z_{1}, \ldots, Z_{r}\right]$ generated by $\left\{Z_{i} \mid i \in I\right\}$. Define the toric orbifold $\mathcal{X}$ as the stack quotient

$$
\mathcal{X}:=\left[U_{\mathcal{A}} / G\right]
$$


where

$$
U_{\mathcal{A}}:=\mathbb{C}^{r} \backslash \bigcup_{I \notin \mathcal{A}} \mathbb{C}^{I}
$$

$\mathcal{X}$ contains the torus $\mathbb{T}:=\widetilde{\mathbb{T}} / G$ as a dense open subset, and the $\widetilde{\mathbb{T}}$-action on $\mathcal{U}_{\Sigma}$ descends to a $\mathbb{T}$-action on $\mathcal{X}$.

2.2. Line bundles and divisors on $\mathcal{X}$. Let $\widetilde{\mathcal{D}}_{i}$ be the $\widetilde{\mathbb{T}}$-divisor in $\mathbb{C}^{r}$ defined by $Z_{i}=0$. Then $\widetilde{\mathcal{D}}_{i} \cap \mathcal{U}_{A}$ descends to a $\mathbb{T}$-divisor $\mathcal{D}_{i}$ in $\mathcal{X}$. We have

$$
\widetilde{M} \cong \operatorname{Pic}_{\widetilde{T}}\left(\mathbb{C}^{r}\right) \cong H_{\widetilde{T}}^{2}\left(\mathbb{C}^{r} ; \mathbb{Z}\right)
$$

where the second isomorphism is given by the $\widetilde{\mathbb{T}}$-equivariant first Chern class $\left(c_{1}\right)_{\widetilde{T}}$. Define 3

$$
\begin{aligned}
& \mathrm{w}_{i}=-\left(c_{1}\right)_{\widetilde{\mathbb{T}}}\left(\mathcal{O}_{\mathbb{C}^{r}}\left(\widetilde{\mathcal{D}}_{i}\right)\right) \in H_{\widetilde{\mathbb{T}}}^{2}\left(\mathbb{C}^{r} ; \mathbb{Z}\right) \cong H_{\mathbb{T}}^{2}\left(\left[\mathbb{C}^{r} / G\right] ; \mathbb{Z}\right) \cong \widetilde{M}, \\
& \bar{D}_{i}^{\mathbb{T}}=\left(c_{1}\right)_{\mathbb{T}}\left(\mathcal{O}_{\mathcal{X}}\left(\mathcal{D}_{i}\right)\right), \quad \bar{D}_{i}^{\widetilde{\mathbb{T}}}=\left(c_{1}\right)_{\widetilde{\mathbb{T}}}\left(\mathcal{O}_{\mathcal{X}}\left(\mathcal{D}_{i}\right)\right) .
\end{aligned}
$$

Then $\left\{-\mathrm{w}_{1}, \ldots,-\mathrm{w}_{r}\right\}$ is a $\mathbb{Z}$-basis of $H_{\widetilde{\pi}}^{2}\left(\mathbb{C}^{r} ; \mathbb{Z}\right) \cong \widetilde{M}$ dual to the $\mathbb{Z}$-basis $\left\{\widetilde{b}_{1}, \ldots, \widetilde{b}_{r}\right\}$ of $\widetilde{N}$. Notice that $\bar{D}_{i}^{\mathbb{T}}=0$ and $\bar{D}_{i}^{\widetilde{\mathbb{T}}}=0$ for $i=r^{\prime}+1, \ldots, r$. We have

$$
\begin{gathered}
\operatorname{Pic}_{\mathbb{T}}(\mathcal{X}) \cong H_{\mathbb{T}}^{2}(\mathcal{X} ; \mathbb{Z}) \cong \widetilde{M} / \oplus_{i=r^{\prime}+1}^{r} \mathbb{Z} D_{i}^{\mathbb{T}} \\
H_{\mathbb{T}}^{2}(\mathcal{X} ; \mathbb{Z})=\bigoplus_{i=1}^{r^{\prime}} \mathbb{Z} \bar{D}_{i}^{\mathbb{T}} \cong \mathbb{Z}^{r^{\prime}}
\end{gathered}
$$

We also have group isomorphisms

$$
\mathbb{L}^{\vee} \cong \operatorname{Pic}_{G}\left(\mathbb{C}^{r}\right) \cong H_{G}^{2}\left(\mathbb{C}^{r} ; \mathbb{Z}\right),
$$

where the second isomorphism is given by the $G$-equivariant first Chern class $\left(c_{1}\right)_{G}$. Notice that a character $\chi \in \mathbb{L}^{\vee}$ defines a line bundle on $\mathcal{X}$

$$
\mathcal{L}_{\chi}=\left\{(z, t) \in \mathcal{U}_{A} \times \mathbb{C} /(z, t) \sim(g \cdot z, \chi(g) \cdot t), g \in G\right\},
$$

where $G$ acts on $\mathcal{U}_{A}$ as a subgroup of $\widetilde{\mathbb{T}}$. There is a canonical $\widetilde{\mathbb{T}}$-action on $\mathcal{L}_{\chi}$ which acts diagonally on $\mathbb{C}^{r}$ and trivially on the fiber. Define

$$
\begin{gathered}
D_{i}=\left(c_{1}\right)_{G}\left(\mathcal{O}_{\mathbb{C}^{r}}\left(\widetilde{\mathcal{D}}_{i}\right)\right) \in H_{G}^{2}\left(\mathbb{C}^{r} ; \mathbb{Z}\right) \cong \mathbb{L}^{\vee}, \\
\bar{D}_{i}=c_{1}\left(\mathcal{O}_{\mathcal{X}}\left(\mathcal{D}_{i}\right)\right) \in H^{2}(\mathcal{X} ; \mathbb{Z}) .
\end{gathered}
$$

We have

$$
\operatorname{Pic}(\mathcal{X}) \cong H^{2}(\mathcal{X} ; \mathbb{Z}) \cong \mathbb{L}^{\vee} / \oplus_{i=r^{\prime}+1}^{r} \mathbb{Z} D_{i}
$$

The map

$$
\bar{\psi}^{\vee}: \operatorname{Pic}_{\mathbb{T}}(\mathcal{X}) \cong H_{\mathbb{T}}^{2}(\mathcal{X} ; \mathbb{Z}) \rightarrow \operatorname{Pic}(\mathcal{X}) \cong H^{2}(\mathcal{X} ; \mathbb{Z})
$$

is descends from $\psi^{\vee}: \operatorname{Pic}_{\widetilde{\mathbb{T}}}\left(\mathbb{C}^{r} ; \mathbb{Z}\right) \cong \widetilde{M} \rightarrow \operatorname{Pic}_{G}\left(\mathbb{C}^{r} ; \mathbb{Z}\right) \cong \mathbb{L}^{\vee}$ and satisfies

$$
\bar{\psi}^{\vee}\left(\bar{D}_{i}^{\mathbb{T}}\right)=\bar{D}_{i} \quad i=1, \ldots, r^{\prime} .
$$

\footnotetext{
${ }^{3}$ The minus sign in the definition of $\mathrm{w}_{i}$ is a convention for the purporse of matching the B-model oscillatory integrals. See Section 4
} 
2.3. Torus invariant subvarieties and their generic stabilizers. Let $\Sigma(d)$ be the set of $d$-dimensional cones. For each $\sigma \in \Sigma(d)$, define

$$
I_{\sigma}=\left\{i \in\{1, \ldots, r\} \mid \rho_{i} \not \sigma\right\} \in \mathcal{A}, \quad I_{\sigma}^{\prime}=\{1, \ldots, r\} \backslash I_{\sigma} .
$$

Let $\tilde{V}(\sigma) \subset U_{\mathcal{A}}$ be the closed subvariety defined by the ideal of $\mathbb{C}\left[Z_{1}, \ldots, Z_{r}\right]$ generated by

$$
\left\{Z_{i}=0 \mid \rho_{i} \subset \sigma\right\}=\left\{Z_{i}=0 \mid i \in I_{\sigma}^{\prime}\right\} .
$$

Then $\mathcal{V}(\sigma):=[\widetilde{V}(\sigma) / G]$ is an $(n-d)$-dimensional $\mathbb{T}$-invariant closed subvariety of $\mathcal{X}=\left[U_{\mathcal{A}} / G\right]$.

Let

$$
G_{\sigma}:=\{g \in G \mid g \cdot z=z \text { for all } z \in \widetilde{V}(\sigma)\} .
$$

Then $G_{\sigma}$ is the generic stabilizer of $\mathcal{V}(\sigma)$. It is a finite subgroup of $G$. If $\tau \subset \sigma$ then $I_{\sigma} \subset I_{\tau}$, so $G_{\tau} \subset G_{\sigma}$. There are two special cases. If $\sigma \in \Sigma(n)$ where $n=\operatorname{dim}_{\mathbb{C}} \mathcal{X}$, then $\mathfrak{p}_{\sigma}:=\mathcal{V}(\sigma)$ is a $\mathbb{T}$ fixed point in $\mathcal{X}$, and $\mathfrak{p}_{\sigma}=B G_{\sigma}$.

2.4. The extended nef cone and the extended Mori cone. In this paragraph, $\mathbb{F}=\mathbb{Q}, \mathbb{R}$, or $\mathbb{C}$. Given a finitely generated free abelian group $\Lambda \cong \mathbb{Z}^{m}$, define $\Lambda_{\mathbb{F}}=\Lambda \otimes_{\mathbb{Z}} \mathbb{F} \cong \mathbb{F}^{m}$. We have the following short exact sequences of vector spaces $(\otimes \mathbb{F}$ with Equation (2) and (4) $)$ :

$$
\begin{aligned}
& 0 \rightarrow \mathbb{L}_{\mathbb{F}} \rightarrow \widetilde{N}_{\mathbb{F}} \rightarrow N_{\mathbb{F}} \rightarrow 0, \\
& 0 \rightarrow M_{\mathbb{F}} \rightarrow \widetilde{M}_{\mathbb{F}} \rightarrow \mathbb{L}_{\mathbb{F}}^{\vee} \rightarrow 0 .
\end{aligned}
$$

Given a maximal cone $\sigma \in \Sigma(n)$, we define

$$
\mathbb{K}_{\sigma}^{\vee}:=\bigoplus_{i \in I_{\sigma}} \mathbb{Z} D_{i} .
$$

Then $\mathbb{K}_{\sigma}^{\vee}$ is a sublattice of $\mathbb{L}^{\vee}$ of finite index. We define the extended nef cone $\widetilde{\operatorname{Nef}_{\mathcal{X}}}$ as below

$$
\widetilde{\operatorname{Nef}}_{\sigma}=\sum_{i \in I_{\sigma}} \mathbb{R}_{\geq 0} D_{i}, \quad \widetilde{\operatorname{Nef}}_{\mathcal{X}}:=\bigcap_{\sigma \in \Sigma(n)} \widetilde{\operatorname{Nef}}_{\sigma} .
$$

The extended $\sigma$-Kähler cone $\widetilde{C}_{\sigma}$ is defined to be the interior of $\widetilde{\mathrm{Nef}}_{\sigma}$; the extended Kähler cone of $\mathcal{X}, \widetilde{C}_{\mathcal{X}}$, is defined to be the interior of the extended nef cone $\widetilde{\mathrm{Nef}}_{\mathcal{X}}$.

Let $\mathbb{K}_{\sigma}$ be the dual lattice of $\mathbb{K}_{\sigma}^{\vee}$; it can be viewed as an additive subgroup of $\mathbb{L}_{\mathbb{Q}}:$

$$
\mathbb{K}_{\sigma}=\left\{\beta \in \mathbb{L}_{\mathbb{Q}} \mid\langle D, \beta\rangle \in \mathbb{Z} \forall D \in \mathbb{K}_{\sigma}^{\vee}\right\},
$$

where $\langle-,-\rangle$ is the natural pairing between $\mathbb{L}_{\mathbb{Q}}^{\vee}$ and $\mathbb{L}_{\mathbb{Q}}$. We have $\mathbb{K}_{\sigma} / \mathbb{L} \cong G$. Define

$$
\mathbb{K}:=\bigcup_{\sigma \in \Sigma(n)} \mathbb{K}_{\sigma} .
$$

Then $\mathbb{K}$ is a subset (which is not necessarily a subgroup) of $\mathbb{L}_{\mathbb{Q}}$, and $\mathbb{L} \subset \mathbb{K}$.

We define the extended Mori cone $\widetilde{\mathrm{NE}}_{\sigma} \subset \mathbb{L}_{\mathbb{R}}$ to be

$$
\widetilde{\mathrm{NE}}_{\mathcal{X}}:=\bigcup_{\sigma \in \Sigma(n)} \widetilde{\mathrm{NE}}_{\sigma}, \quad \widetilde{\mathrm{NE}}_{\sigma}=\left\{\beta \in \mathbb{L}_{\mathbb{R}} \mid\langle D, \beta\rangle \geq 0 \forall D \in \widetilde{\mathrm{Nef}}_{\sigma}\right\} .
$$

Finally, we define extended curve classes

$$
\mathbb{K}_{\mathrm{eff}, \sigma}:=\mathbb{K}_{\sigma} \cap \widetilde{\mathrm{NE}_{\sigma}}, \quad \mathbb{K}_{\mathrm{eff}}:=\mathbb{K} \cap \widetilde{\mathrm{NE}}(\mathcal{X})=\bigcup_{\sigma \in \Sigma(n)} \mathbb{K}_{\mathrm{eff}, \sigma} .
$$


Assumption 2.1 (semi-positive (Weakly Fano) condition). From now on, we assume that we may choose $b_{r^{\prime}+1}, \ldots, b_{r}$ such that $\hat{\rho}:=D_{1}+\cdots+D_{r}$ is contained in the closure of the extended Kähler cone $\widetilde{C}_{\mathcal{X}}$.

Remark 2.2. We make the above assumption so that the equivariant mirror theorem in [6, 7] has an explicit mirror map.

2.5. The inertia stack and the Chen-Ruan cohomology. Given $\sigma \in \Sigma$, define

$$
\operatorname{Box}(\sigma):=\left\{v \in N: v=\sum_{i \in I_{\sigma}^{\prime}} c_{i} b_{i}, \quad 0 \leq c_{i}<1\right\} .
$$

If $\tau \subset \sigma$ then $I_{\tau}^{\prime} \subset I_{\sigma}^{\prime}$, so $\operatorname{Box}(\tau) \subset \operatorname{Box}(\sigma)$.

Given a real number $x$, let $\lfloor x\rfloor$ be the greatest integer less than or equal to $x,\lceil x\rceil$ be the least integer greater or equal to $x$, and $\{x\}=x-\lfloor x\rfloor$ is the fractional part of $x$. Define $v: \mathbb{K}_{\sigma} \rightarrow N$ by

$$
v(\beta)=\sum_{i=1}^{r}\left\lceil\left\langle D_{i}, \beta\right\rangle\right\rceil b_{i}=\sum_{i \in I_{\sigma}^{\prime}}\left\{-\left\langle D_{i}, \beta\right\rangle\right\} b_{i},
$$

so $v(\beta) \in \operatorname{Box}(\sigma)$. Indeed, $v$ induces a bijection $\mathbb{K}_{\sigma} / \mathbb{L} \cong \operatorname{Box}(\sigma)$.

For any $\tau \in \Sigma$ there exists $\sigma \in \Sigma(n)$ such that $\tau \subset \sigma$. The bijection $G_{\sigma} \rightarrow \operatorname{Box}(\sigma)$ restricts to a bijection $G_{\tau} \rightarrow \operatorname{Box}(\tau)$. Define

$$
\operatorname{Box}(\boldsymbol{\Sigma}):=\bigcup_{\sigma \in \Sigma} \operatorname{Box}(\sigma)=\bigcup_{\sigma \in \Sigma(n)} \operatorname{Box}(\sigma) .
$$

There is a bijection $\mathbb{K} / \mathbb{L} \rightarrow \operatorname{Box}(\boldsymbol{\Sigma})$.

Given $v \in \operatorname{Box}(\sigma)$, where $\sigma \in \Sigma(d)$, define $c_{i}(v) \in[0,1) \cap \mathbb{Q}$ by

$$
v=\sum_{i \in I_{\sigma}^{\prime}} c_{i}(v) b_{i} .
$$

Suppose that $k \in G_{\sigma}$ corresponds to $v \in \operatorname{Box}(\sigma)$ under the bijection $G_{\sigma} \cong \operatorname{Box}(\sigma)$, then

$$
\chi_{i}(k)= \begin{cases}1, & i \in I_{\sigma}, \\ e^{2 \pi \sqrt{-1} c_{i}(v)}, & i \in I_{\sigma}^{\prime} .\end{cases}
$$

Define

$$
\operatorname{age}(k)=\operatorname{age}(v)=\sum_{i \notin I_{\sigma}} c_{i}(v) .
$$

Let $I U=\left\{(z, k) \in U_{\mathcal{A}} \times G \mid k \cdot z=z\right\}$, and let $G$ acts on $I U$ by $h \cdot(z, k)=(h \cdot z, k)$. The inertia stack $\mathcal{I} \mathcal{X}$ of $\mathcal{X}$ is defined to be the quotient stack

$$
\mathcal{I X}:=[I U / G] \text {. }
$$

The inertial stack $\mathcal{I X}$ comes with a projection map pr: $\mathcal{I} \mathcal{X} \rightarrow \mathcal{X}$. Note that $\left(z=\left(Z_{1}, \ldots, Z_{r}\right), k\right) \in I U$ if and only if

$$
k \in \bigcup_{\sigma \in \Sigma} G_{\sigma} \text { and } Z_{i}=0 \text { whenever } \chi_{i}(k) \neq 1 .
$$

So

$$
I U=\bigcup_{v \in \operatorname{Box}(\boldsymbol{\Sigma})} U_{v}
$$

where

$$
U_{v}:=\left\{\left(Z_{1}, \ldots, Z_{m}\right) \in U_{\mathcal{A}}: Z_{i}=0 \text { if } c_{i}(v) \neq 0\right\} .
$$


The connected components of $\mathcal{I X}$ are

$$
\left\{\mathcal{X}_{v}:=\left[U_{v} / G\right]: v \in \operatorname{Box}(\boldsymbol{\Sigma})\right\} .
$$

The involution $I U \rightarrow I U,(z, k) \mapsto\left(z, k^{-1}\right)$ induces involutions inv $: \mathcal{I X} \rightarrow \mathcal{I X}$ and inv : $\operatorname{Box}(\boldsymbol{\Sigma}) \rightarrow \operatorname{Box}(\boldsymbol{\Sigma})$ such that $\operatorname{inv}\left(\mathcal{X}_{v}\right)=\mathcal{X}_{\operatorname{inv}(v)}$. Define the $\mathbb{T}$-fixed point $\mathfrak{p}_{\sigma, v}=\operatorname{pr}^{-1}\left(\mathfrak{p}_{\sigma}\right) \cap \mathcal{X}_{v}$.

In the remainder of this subsection, we consider rational cohomology, and write $H^{*}(-)$ instead of $H^{*}(-; \mathbb{Q})$. The Chen-Ruan orbifold cohomology, as a vector space, is defined to be 5,42 .

$$
H_{\mathrm{CR}}^{*}(\mathcal{X})=\bigoplus_{v \in \operatorname{Box}(\boldsymbol{\Sigma})} H^{*}\left(\mathcal{X}_{v}\right)[2 \operatorname{age}(v)] .
$$

Denote $\mathbf{1}_{v}$ to be the unit in $H^{*}\left(\mathcal{X}_{v}\right)$. Then $\mathbf{1}_{v} \in H_{\mathrm{CR}}^{2 \text { age }(v)}(\mathcal{X})$. In particular,

$$
H_{\mathrm{CR}}^{0}(\mathcal{X})=\mathbb{Q} \mathbf{1}_{0} \text {. }
$$

Since $\mathcal{X}$ is proper, the orbifold Poincaré pairing on $H_{\mathrm{CR}}^{*}(\mathcal{X})$ is defined as

$$
(\alpha, \beta):=\int_{\mathcal{I X}} \alpha \cdot \operatorname{inv}^{*}(\beta),
$$

We also have an equivariant pairing on $H_{\mathrm{CR}, \mathbb{T}}^{*}(\mathcal{X})$ :

$$
(\alpha, \beta)_{\mathbb{T}}:=\int_{\mathcal{I X}_{\mathbb{T}}} \alpha \cdot \operatorname{inv}^{*}(\beta),
$$

where

$$
\int_{\mathcal{I} \mathcal{X}_{\mathbb{T}}}: H_{\mathrm{CR}, \mathbb{T}}^{*}(\mathcal{X}) \rightarrow H_{\mathbb{T}}^{*}(\text { point })=H^{*}(B \mathbb{T})
$$

is the equivariant pushforward to a point. Here the dot - (sometimes omitted) is the usual product in $H^{*}(\mathcal{I} \mathcal{X})$ as the same vector space as $H_{\mathrm{CR}}^{*}(\mathcal{X})$. The product in the Chen-Ruan cohomology, as defined in [5] or for many cases [42], does not explicitly appear in this paper.

\section{THE MIRROR OF A TORIC ORBIFOLD}

3.1. Coherent-constructible correspondence and HMS: a quick review. The equivariant mirror to $\mathcal{X}$ is a Landau-Ginzburg model $\left(\left(\mathbb{C}^{*}\right)^{n}, W\right)$, where $W$ : $\left(\mathbb{C}^{*}\right)^{n} \rightarrow \mathbb{C}$ is the holomorphic superpotential function. To consider the equivariant information on $\mathcal{X}$, one needs to consider the universal cover of $\left(\mathbb{C}^{*}\right)^{n}$. As a symplectic manifold, this $\left(\mathbb{C}^{*}\right)^{n}$ is naturally identified with $\mathcal{Y}=T^{*}\left(M_{\mathbb{R}} / M\right) \cong T^{*}\left(S^{1}\right)^{n}$, and its universal cover $\mathbb{C}^{n}$ is identified with $\widetilde{\mathcal{Y}}=T^{*} M_{\mathbb{R}}=N_{\mathbb{R}} \times M_{\mathbb{R}}$.

For any $\tau \in \Sigma$, let $N_{\tau}$ be the sublattice of $N$ generated by $b_{i} \in \tau$, and $M_{\tau}=$ $\operatorname{Hom}\left(N_{\tau}, \mathbb{Z}\right)$. Define $\tau_{\underline{\chi}}^{\perp}=\left\{u \in M_{\mathbb{R}} \mid\langle v, u\rangle=\underline{\chi}(v), v \in \tau\right\}$.

Definition 3.1. Let $U$ be a real manifold. We say a set in $T^{*} U$ is conical if it is invariant under the dilation $(v, u) \mapsto(t v, u)$ where $u \in U, v \in T_{v}^{*} U$ and $t \in \mathbb{R}_{>0}$.

The stacky fan $\boldsymbol{\Sigma}$ defines the following conical Lagrangian in $T^{*} M_{\mathbb{R}}=N_{\mathbb{R}} \times M_{\mathbb{R}}$.

$$
\Lambda_{\Sigma}=\bigcup_{\tau \in \Sigma} \bigcup_{\underline{\chi} \in M_{\tau}}(-\tau) \times \tau_{\underline{\chi}}^{\perp},
$$

We embed $\rho: T^{*} M_{\mathbb{R}} \hookrightarrow \overline{T^{*} M_{\mathbb{R}}}=\left\{(v, u) \in T^{*} M_{\mathbb{R}} \mid\|v\| \leq 1\right\}$ by

$$
(v, u) \mapsto\left(\frac{v}{\sqrt{1+\|v\|^{2}}}, u\right) .
$$


Here $v \in N_{\mathbb{R}}$ and $u \in M_{\mathbb{R}}$. The infinity part of $\Lambda_{\Sigma}^{\infty}=\overline{\Lambda_{\Sigma}} \backslash \Lambda_{\Sigma}$ is piecewise Legendrian in the contact boundary unit sphere bundle $T^{\infty} M_{\mathbb{R}}=\overline{T^{*} M_{\mathbb{R}}} \backslash T^{*} M_{\mathbb{R}}$.

We refer the reader to 9 11] for the details of homological mirror symmetry, and only recall the relevant parts here. Let $D^{b} \mathrm{Coh}_{\mathbb{T}}(\mathcal{X})$ be the derived category of equivariant coherent sheaves on $\mathcal{X}$, and let $D^{b} \mathrm{Sh}_{c c}\left(M_{\mathbb{R}} ; \Lambda_{\Sigma}\right)$ be the derived category of compactly supported constructible sheaves on $M_{\mathbb{R}}$ whose singular supports are subsets of $\Lambda_{\boldsymbol{\Sigma}}$. Define $\operatorname{Fuk}\left(T^{*} M_{\mathbb{R}} ; \Lambda_{\boldsymbol{\Sigma}}\right)$ be the derived zunwrapped Fukaya category (in the sense of $[34,35]$ ) of $T^{*} M_{\mathbb{R}}$ of horizontally compactly supported Lagrangian branes $L$ such that their infinity parts $L^{\infty} \subset \Lambda_{\Sigma}^{\infty}$. The homological mirror symmetry of Fang-Liu-Treumann-Zaslow [9,10] could be summarized in the following diagram of quasi-equivalence functors.

Theorem 3.2. There is an exact quasi-equivalent functor $\kappa$ as in the following diagram. Combined with the microlocalization functor $\mu$, which is also exact and an equivalence (34,35), $\mu \circ \kappa$ is a version of homological mirror symmetry $-\mu \circ \kappa(\mathcal{L})$ is isomorphic to the $S Y Z$ transformation of $\mathcal{L}$, which takes an equivariant line bundle and produces a Lagrangian in $\operatorname{Fuk}\left(T^{*} M_{\mathbb{R}} ; \Lambda_{\boldsymbol{\Sigma}}\right)$ as given in [2,30].

$$
D^{b} \operatorname{Coh}_{\mathbb{T}}(\mathcal{X}) \stackrel{\kappa}{\longrightarrow} D^{b} \operatorname{Sh}_{c c}\left(M_{\mathbb{R}} ; \Lambda_{\Sigma}\right) \stackrel{\mu}{\longrightarrow} \operatorname{Fuk}\left(T^{*} M_{\mathbb{R}} ; \Lambda_{\Sigma}\right) .
$$

Here we only explain the functor $\kappa$ and $\mu \circ \kappa$ on the object level. Let $\vec{l}=$ $\left(l_{1}, \ldots, l_{r^{\prime}}\right) \in \mathbb{Z}^{r^{\prime}}$. We say a line bundle $\mathcal{L}$ is $\mathbb{Q}$-ample if certain postive powers of it is a pullback of an ample line bundle on the coarse moduli $X$. Given a $\mathbb{T}$ equivariant $\mathbb{Q}$-ample line bundle $\mathcal{L}_{\vec{l}}=\mathcal{O}_{\mathcal{X}}\left(\sum_{i=1}^{r^{\prime}} l_{i} \mathcal{D}_{i}\right)$ on $\mathcal{X}$, one can associate its moment polytope

$$
\Delta_{\vec{l}}=\left\{u \in M_{\mathbb{R}} \mid\left\langle u, b_{i}\right\rangle>-l_{i}\right\} .
$$

The $\mathbb{Q}$-ampleness ensures that $\Delta_{\vec{l}}$ is a convex open polytope. The functor $\kappa$ is characterized by mapping the $\mathbb{Q}$-ample line bundle $\mathcal{L}_{\vec{l}}$ to $i_{\Delta_{\vec{l}}}$ ! $\omega_{\Delta_{\vec{l}}}$, where $i_{\Delta_{\vec{l}}}: \Delta_{\vec{l}} \hookrightarrow$ $M_{\mathbb{R}}$ is the embedding and $\omega_{\Delta_{\vec{l}}}$ is the Verdier dual of the constant sheaf $\mathbb{C}_{\Delta_{\vec{l}}}$ on $\Delta_{\vec{l}}$. The constructible sheaf $i_{\Delta_{\vec{l}} *} \mathbb{C}_{\Delta_{\vec{l}}}$ is called the standard sheaf over $\Delta_{\vec{l}}$, while its Verdier dual $i_{\Delta_{l} !} \omega_{\Delta_{l}}$ is called costandard sheaf. The microlocalization functor $\mu$ takes the standard sheaf $i_{\Delta_{\vec{l}} *} \mathbb{C}_{\Delta_{\vec{l}}}$ to the graph of $d \log m_{\Delta_{\vec{l}}}$, where $m_{\Delta_{\vec{l}}}$ is a function on $\bar{\Delta}_{\vec{l}}$ with $\left.m_{\Delta_{\vec{l}}}\right|_{\partial \Delta_{\vec{l}}}=0$ and $\left.m_{\Delta_{\vec{l}}}\right|_{\Delta_{\vec{l}}}>0$. This graph $d \log m_{\Delta_{\vec{l}}}$ is a standard Lagrangian. The functor also takes the costandard sheaf $i_{\Delta_{\vec{l}} !} \omega_{\Delta_{\vec{l}}}$ to a costandard Lagrangian which is the graph of $-d \log m_{\Delta_{\vec{l}}}$.
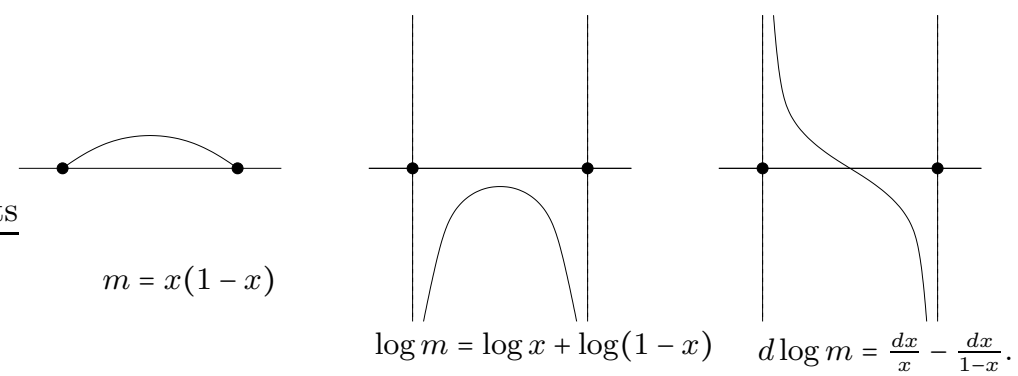

Figure 1. The graphs of $m, \log m$ and $d \log m$ of an open interval $U \subset \mathbb{R}$. The graph of $d \log m$ is the standard Lagrangian over $U$. If $\mathcal{X}=\mathbb{P}^{1}$, an ample line bundle $\mathcal{O}_{\mathbb{P}^{1}}\left(a \mathcal{D}_{1}+b \mathcal{D}_{2}\right)$ corresponds to the costandard sheaf over $(-a, b)$ under $\kappa$. 
3.2. Landau-Ginzburg B-model and oscillatory integrals. The symplectic structure on $T^{*} M_{\mathbb{R}}$ only tackles the B-model information on $\mathcal{X}$. In order to make predictions on the Gromov-Witten invariants on $\mathcal{X}$ one needs to consider the complex structure on the equivariant mirror Landau-Ginzburg model of $\mathcal{X}$. In this subsection, we first define such a Landau-Ginzburg model from the viewpoint of complex geometry, which should be mirror to the $A$-model of $\mathcal{X}$, and then identify it with $T^{*} M_{\mathbb{R}}=N_{\mathbb{R}} \times M_{\mathbb{R}}$.

We fix an integral basis $e_{1}, \ldots, e_{k} \in \mathbb{L}$ and its dual basis $e_{1}^{\vee}, \ldots, e_{k}^{\vee}$ in $\mathbb{L}^{\vee}$. We require that each $e_{a}^{\vee}$ is in $\widetilde{\mathrm{Nef}_{\mathcal{X}}}$, and $e_{k^{\prime}}^{\vee}, \ldots, e_{k}^{\vee}$ are in $\sum_{i=r^{\prime}+1}^{r} \mathbb{R}_{\geq 0} D_{i}$. Define the charge vectors

So

$$
l^{(a)}=\left(l_{1}^{(a)}, \ldots, l_{r}^{(a)}\right) \in \mathbb{Z}^{r}, \quad \psi\left(e_{a}\right)=\sum_{i=1}^{r} l_{i}^{(a)} \widetilde{b}_{i}
$$

$$
D_{i}=\psi^{\vee}\left(D_{i}^{\mathbb{T}}\right)=\sum_{a=1}^{k} l_{i}^{(a)} e_{a}^{\vee}, \quad i=1, \ldots, r .
$$

Define the Landau-Ginzburg B-model as follows

$$
\mathcal{Y}_{q}=\left\{\left(X_{1}, \ldots, X_{r}\right) \in\left(\mathbb{C}^{*}\right)^{r} \mid \prod_{i=1}^{r} X_{i}^{l_{i}^{(a)}}=q_{a}, a=1, \ldots, k\right\} .
$$

Here $q_{1}, \ldots, q_{k}$ are complex parameters. We assume $q_{a}>0$ for all $a$. Apply the exact functor $\operatorname{Hom}\left(-, \mathbb{C}^{*}\right)$ to the short exact sequence (2) and we get

$$
1 \rightarrow \operatorname{Hom}\left(N, \mathbb{C}^{*}\right) \rightarrow\left(\mathbb{C}^{*}\right)^{r} \stackrel{\mathfrak{p}}{\longrightarrow} \mathcal{M}=\operatorname{Hom}\left(\mathbb{L}, \mathbb{C}^{*}\right) \rightarrow 1 .
$$

We see that $\mathcal{Y}_{q}=\mathfrak{p}^{-1}(q) \cong\left(\mathbb{C}^{*}\right)^{k}$ is a subtorus in $\left(\mathbb{C}^{*}\right)^{r}$. Here $q=\left(q_{1}, \ldots, q_{k}\right)$ are coordinates on $\mathcal{M}$. For any $\beta \in \mathbb{K}$, denote $q^{\beta}=\prod_{a=1}^{k} q_{a}^{\left\langle\beta, e_{a}^{\vee}\right\rangle}$. This notion may involve factional powers as $\beta \in \mathbb{K}$ in which $\mathbb{L}$ is a sublattice.

Let $u_{1}, \ldots, u_{n}$ be the coordinates on $M_{\mathbb{R}}$ and $v_{1}, \ldots, v_{n}$ be the coordinates on $N_{\mathbb{R}}$. Let $y_{i}=-v_{i}+2 \pi \sqrt{-1} u_{i}$ and $Y_{i}=e^{y_{i}}$. Then $y_{1}, \ldots, y_{n}$ are complex coordinates on $\widetilde{\mathcal{Y}}=N_{\mathbb{R}} \times M_{\mathbb{R}}$, while $Y_{1}, \ldots, Y_{n}$ are complex coordinates on $\mathcal{Y}=N_{\mathbb{R}} \times M_{\mathbb{R}} / M \cong$ $T^{*}\left(M_{\mathbb{R}} / M\right)$.

We fix a splitting of the exact sequence (2) over rational numbers, i.e. we choose a surjective map $\eta: \widetilde{N}_{\mathbb{Q}} \rightarrow \mathbb{L}_{\mathbb{Q}}$ such that $\eta\left(\widetilde{b}_{i}\right)=\sum_{a=1}^{k} \eta_{i a} e_{a}\left(\right.$ so $\left.e_{a}^{\vee}=\sum_{i=1}^{r} \eta_{i a} D_{i}\right)$ and $\psi \circ \eta=$ id, where $\eta_{i a} \in \mathbb{Q}$. This splitting identifies $\mathcal{Y}_{q}$ with $\mathcal{Y}=\operatorname{Hom}\left(N, \mathbb{C}^{*}\right) \cong$ $N_{\mathbb{R}} \times\left(M_{\mathbb{R}} / M\right)$ as follows.

$$
X_{i}=q_{i}^{\prime} Y^{b_{i}}, \quad Y^{b_{i}}=\prod_{j=1}^{n} Y_{j}^{b_{i j}}, \quad q_{i}^{\prime}=\prod_{a=1}^{k} q_{a}^{\eta_{i a}} .
$$

Here $b_{i}=\left(b_{i 1}, \ldots, b_{i n}\right)$ is the coordinate of $b_{i}$ in $N$. We also identifies $\widetilde{\mathcal{Y}}_{q}$ with $\widetilde{\mathcal{Y}}=N_{\mathbb{R}} \times M_{\mathbb{R}}$. We choose $q_{i}^{\prime}>0$ as they may involve fractional powers of $q_{a}$ in (8).

Definition 3.3. The superpotential on $\mathcal{Y}_{q}$ is

$$
W=\sum_{i=1}^{r}\left(t_{0}+X_{i}\right)
$$

where $t_{0} \in \mathbb{C}$ is a parameter which plays the role of $H^{0}$-part on the A-side under the mirror relation. This superpotential depends on $t_{0}$ and $q$. We let $q_{0}=e^{t_{0}}$. The 
equivariant superpotential on $\widetilde{\mathcal{Y}}_{q}$ is

$$
\widetilde{W}=W+\sum_{i=1}^{r} w_{i} x_{i}
$$

Here $x_{i}=\log X_{i}$, and the equivariant parameters $w_{i} \in \mathbb{C}$.

Once we choose

$$
x_{i}=\sum_{a=1}^{k} \eta_{i a} \log q_{a}+\sum_{j=1}^{n} b_{i j} y_{j}
$$

the equivariant superpotential $\widetilde{W}$ is well-defined on $\widetilde{\mathcal{Y}}_{q}$. It depends on $t_{0}, \log q_{a}$ (we can choose them in $\mathbb{R}$ since all $q_{a}>0$ ) and $w_{i}$.

The following holomorphic form on $\widetilde{\mathcal{Y}}=N_{\mathbb{R}} \times M_{\mathbb{R}}$

$$
\Omega=\frac{d Y_{1} \ldots d Y_{n}}{Y_{1} \ldots Y_{n}}
$$

is also a holomorphic form on $\widetilde{\mathcal{Y}}_{q}$ once we identify $\widetilde{\mathcal{Y}}_{q}$ with $\widetilde{\mathcal{Y}}$. Let $W_{\eta}$ and $\widetilde{W}_{\eta}$ be the function $W$ and $\widetilde{W}$ on $\mathcal{Y}$ and $\widetilde{\mathcal{Y}}$ respectively once we fix $\eta$. Consider a partial compactification $\left(\overline{T^{*} M_{\mathbb{R}}}\right)_{\Lambda_{\Sigma}}=T^{*} M_{\mathbb{R}} \cup \Lambda_{\Sigma}^{\infty} \subset \overline{T^{*} M_{\mathbb{R}}}$. For any $z>0$ and

$$
\Xi \in H_{n}\left(\left(\overline{T^{*} M_{\mathbb{R}}}\right)_{\Lambda_{\Sigma}}, \Lambda_{\Sigma}^{\infty} ; \mathbb{Z}\right)
$$

we define the integral

$$
I_{\Xi}:=\int_{\Xi} e^{-\frac{\widetilde{W}_{\eta}}{z}} \Omega
$$

This definition a priori depends on the choice of $\eta$, which gives the identification of $\widetilde{\mathcal{Y}}_{q}$ with $\widetilde{\mathcal{Y}}=N_{\mathbb{R}} \times M_{\mathbb{R}}$. We have the following two propositions.

Proposition 3.4. The integral $I_{\Xi}$ converges for any $\Xi \in H_{n}\left(\left(\overline{T^{*} M_{\mathbb{R}}}\right)_{\Lambda_{\Sigma}}, \Lambda_{\Sigma}^{\infty} ; \mathbb{Z}\right)$ and any choice of $\eta$.

Proof. For each $\tau \in \Sigma$ where $\tau$ is spanned by $\rho_{i}, i \in I_{\tau}^{\prime}$, the linear Lagrangian

$$
\Lambda_{\tau}=\bigcup_{\chi \in M_{\tau}}(-\tau) \times \tau_{\chi}^{\perp}
$$

is characterized by

$$
\begin{gathered}
\operatorname{Re}\left(y^{b_{i}}\right)=-\sum_{j=1}^{n} b_{i j} v_{j} \geq 0, \quad \operatorname{Im}\left(y^{b_{i}}\right)=2 \pi \sqrt{-1} \sum_{j=1}^{n} b_{i j} u_{j} \in 2 \pi \sqrt{-1} \mathbb{Z}, \quad i \in I_{\tau}^{\prime} ; \\
\operatorname{Re}\left(y^{b_{i}}\right)=0, \quad i \in I_{\tau} .
\end{gathered}
$$

We view $T^{*} M_{\mathbb{R}}=N_{\mathbb{R}} \times M_{\mathbb{R}}$ as the interior part of the unit ball bundle $\overline{T^{*} M_{\mathbb{R}}}$ under the embedding map $\rho$. Then $\rho_{*} e^{-\frac{\widetilde{W}}{z}}$ is an analytic function on $\rho\left(T^{*} M_{\mathbb{R}}\right)$, and since $z>0$ it exponentially decays to zero near $\Lambda_{\tau}^{\infty}$ - it can be extended to a neighborhood $\tilde{\Lambda}_{\tau}^{\infty}$ of $\Lambda_{\tau}^{\infty} \subset \overline{T^{*} M_{\mathbb{R}}}$ for each $\tau$, with value 0 on $\tilde{\Lambda}_{\tau}^{\infty} \backslash \rho\left(T^{*} M_{\mathbb{R}}\right)$. The differential form $\rho_{*}\left(e^{-\frac{\widetilde{W}}{z}} \Omega\right)$ can also be defined on this neighborhood. Thus denote

$$
\left(\overline{T^{*} M_{\mathbb{R}}}\right)_{\Lambda_{\Sigma}}=T^{*} M_{\mathbb{R}} \cup \bigcup_{\tau \in \Sigma} \tilde{\Lambda}_{\tau}^{\infty} .
$$

The differential form $e^{-\frac{\widetilde{W}}{z}} \Omega$ is a closed analytic form on this $\left(\overline{T^{*} M_{\mathbb{R}}}\right)_{\Lambda_{\Sigma}} \subset \overline{T^{*} M_{\mathbb{R}}}$, and vanishes on the infinity boundary. 
Thus the integral can be evaluated as

$$
I_{\Xi}=\int_{\overline{\rho(\Xi)}} \rho_{*}\left(e^{-\frac{\widetilde{W}}{z}} \Omega\right) .
$$

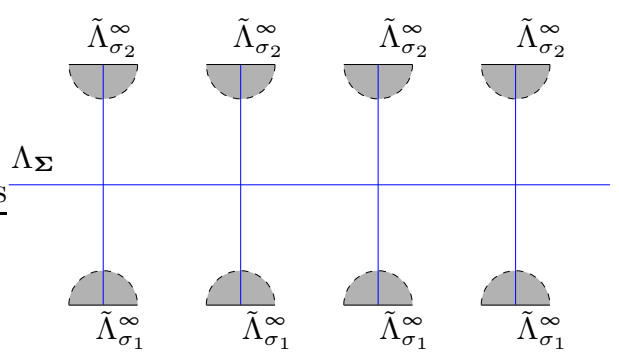

Figure 2. $\Lambda_{\Sigma}$ for $\mathbb{P}^{1}$ (blue fishbone). Shaded half disks are $\tilde{\Lambda}_{\sigma}^{\infty}$ on which $\widetilde{W}$ can be extended. Here two 1-cones of $\mathbb{P}^{1}$ are $\sigma_{1}=\mathbb{R}_{\geq 0}$ and $\sigma_{2}=\mathbb{R}_{\leq 0}$.

Proposition 3.5. The integral $I_{\Xi}$ is does not depend on the choice of $\eta$.

Proof. Let $\eta_{1}$ and $\eta_{2}$ be two splittings of the exact sequence (2). Then we have two identifications of $N_{\mathbb{R}} \times M_{\mathbb{R}}$ with $\widetilde{\mathcal{Y}}_{q}$, and two sets of coordiantes $\left\{y_{i}^{1}\right\}$ and $\left\{y_{i}^{2}\right\}$ on $\widetilde{\mathcal{Y}}_{q}$. By (8) and the fact $\eta_{i a} \in \mathbb{Q}$, they are related by

$$
y_{i}^{1}=y_{i}^{2}+c_{i}, c_{i} \in \mathbb{R} \text {. }
$$

The Calabi-Yau form $\Omega$ is invariant under this change of variables, and the cycle $\Xi$ is also invariant (they are related by a homotopy $y_{i}^{1}=y_{i}^{2}+t c_{i}, t \in[0,1]$, which preserves $\Lambda_{\Sigma}^{\infty}$ ).

Given a sheaf $E \in \operatorname{Sh}_{c c}\left(M_{\mathbb{R}} ; \Lambda_{\Sigma}\right)$, let

$$
\mathrm{CC}(E) \in H_{S S(E)}^{0}\left(M_{\mathbb{R}} ; \pi^{-1} \omega_{M_{\mathbb{R}}}\right) \subset H_{\Lambda_{\Sigma}}^{0}\left(M_{\mathbb{R}} ; \pi^{-1} \omega_{M_{\mathbb{R}}}\right)
$$

be its characteristic cycle. This cycle represents an element in $H_{n}\left(\left(\overline{T^{*} M_{\mathbb{R}}}\right)_{\Lambda_{\Sigma}}, \Lambda_{\Sigma}^{\infty} ; \mathbb{Z}\right)$ since $\operatorname{SS}(E) \subset \Lambda_{\Sigma}^{\infty}$. We still denote it by $\mathrm{CC}(E)$ by a slight abuse of notation. For any coherent sheaf $\mathcal{L} \in \operatorname{Coh}_{\mathbb{T}}(\mathcal{X})$, define its mirror cycle to be

$$
\Xi(\mathcal{L}):=\mathrm{CC}(\kappa(\mathcal{L})) .
$$

We define the central charge of $E$ as follows.

Definition 3.6 (A and B-model central charge).

$$
\begin{aligned}
& I_{B}(E)=I_{\mathrm{CC}(E)}=\int_{\mathrm{CC}(E)} e^{-\frac{\widetilde{W}}{z}} \Omega, \\
& I_{A}(\mathcal{L})=I_{\Xi(\mathcal{L})}=\int_{\mathrm{CC}(\kappa(\mathcal{L}))} e^{-\frac{\widetilde{W}}{z}} \Omega .
\end{aligned}
$$

Proposition 3.7. If $E \rightarrow F \rightarrow G \rightarrow E[1]$ is an exact triangle, then

$$
I_{B}(F)=I_{B}(E)+I_{B}(G) .
$$

Proof. It follows from $\mathrm{CC}(F)=\mathrm{CC}(E)+\mathrm{CC}(G)$ [23, Proposition 9.4.5]. 
We give a description of the characteristic cycle of the constructible sheaf corresponding to a $\mathbb{Q}$-ample line bundle. Let $\mathcal{L}_{\vec{l}}$ be a $\mathbb{Q}$-ample line bundle. For each cone $\tau \in \Sigma$ spanned by $b_{i} \in \tau$, it determines a face $\Delta_{\tau}(\vec{l}) \subset \Delta_{\vec{l}} \subset M_{\mathbb{R}}$

$$
\Delta_{\tau}(\vec{l})=\left\{u \in M_{\mathbb{R}} \mid\left\langle b_{i}, u\right\rangle \geq-l_{i}, \forall b_{i} \notin \tau ; \quad\left\langle b_{i}, u\right\rangle=-l_{i}, \forall b_{i} \in \tau\right\} .
$$

In particular, each top dimensional cone $\sigma$ determines a vertex $\underline{\chi}_{\sigma}(\vec{l})=\Delta_{\sigma}(\vec{l})$ of $\Delta_{\vec{l}}=\Delta_{\{0\}}(\vec{l}) \subset M_{\mathbb{R}}$. Then $\Xi\left(\mathcal{L}_{\vec{l}}\right)$ is the following

$$
\Xi\left(\mathcal{L}_{\vec{l}}\right)=\mathrm{CC}\left(\kappa\left(\mathcal{L}_{\vec{l}}\right)\right)=\mathrm{CC}\left(i_{\Delta_{\vec{l}} !} \omega_{\Delta_{\vec{l}}}\right)=\sum_{\tau \in \Sigma}(-\tau) \times \Delta_{\tau}(\vec{l}) .
$$

Remark 3.8. When $\mathcal{L}_{\vec{l}}$ is an ample line bundle on a smooth complete toric manifold $\mathcal{X}$, one can follow the SYZ T-duality procedure as prescribed in 2, 30. We recall briefly what this procedure is here, and refer to [10] for more details in the toric case.

Let $\mathcal{X}_{0}=\mathcal{X} \backslash \mathcal{D}_{\infty}$, where $\mathcal{D}_{\infty}=\sum_{i=1}^{r} \mathcal{D}_{i}$ is an anti-canonical divisor. Let $s_{\vec{l}}$ be a meromorphic section such that $\left(s_{\vec{l}}\right)=\sum_{i=1}^{r} l_{i} \mathcal{D}_{i}$. It is holomorphic on $\mathcal{X}_{0}$. A $\mathbb{T}_{\mathbb{R}}$-invariant hermitian metric $h$ on the line bundle $\mathcal{L}_{\vec{l}}$ defines the norm $\left\|s_{\vec{l}}\right\|_{h}$ as $\mathbb{T}_{\mathbb{R}}$-invariant function on $\mathcal{X}_{0}$. Let $f_{\vec{l}}=-\log \left\|s_{\vec{l}}\right\|_{h}$. Identify $\mathcal{X}_{0}=N \otimes_{\mathbb{Z}} \mathbb{C}^{*}$, where $v_{1}, \ldots, v_{n} \in \mathbb{R}$ and $\theta_{1}, \ldots, \theta_{n} \in \mathbb{R} /(2 \pi \mathbb{Z})$ are coordinates on $\mathcal{X}_{0}$ such that they give complex coordinates $e^{v_{i}+\sqrt{-1} \theta_{i}}$. The $\mathbb{T}_{\mathbb{R}}$-invariance of $f_{\vec{l}}$ implies $f_{\vec{l}}$ is a function of $v$ only. Define the SYZ dual brane by

$$
\operatorname{SYZ}\left(\mathcal{L}_{\vec{l}}\right)=\left\{(u, v) \in M_{\mathbb{R}} \times N_{\mathbb{R}}: u_{j}=\frac{\partial f_{\vec{l}, h}}{\partial v_{j}}, j=1, \ldots, n\right\} \subset \widetilde{\mathcal{Y}}
$$

The resulting submanifold, as shown in [10, is a costandard Lagrangian on the open set $\Delta_{\vec{l}}$ when $\mathcal{L}_{\vec{l}}$ is ample. The results are isomorphic in the Fukaya category for different choices of $h$. Moreover, for any costandard sheaf $\mathcal{F}$, the result of 38 states the characteristic cycle is the conical limit of the costandard Lagrangian $\mu(\mathcal{F})$

$$
\mathrm{CC}(\mathcal{F})=\lim _{\epsilon \rightarrow 0} \epsilon \mu(\mathcal{F})
$$

The integral on $\epsilon \mathrm{SYZ}\left(\mathcal{L}_{\vec{l}}\right)$

$$
\int_{\epsilon \mathrm{SYZ}\left(\mathcal{L}_{\vec{l}}\right)} e^{-\frac{\widetilde{W}}{z}} \Omega
$$

remains unchanged as $\epsilon \rightarrow 0$ since $\epsilon \operatorname{SYZ}\left(\mathcal{L}_{\vec{l}}\right)=\epsilon \mu \circ \kappa\left(\mathcal{L}_{\vec{l}}\right)$ corresponds to the same cycle in $H_{n}\left(\left(\overline{T^{*} M_{\mathbb{R}}}\right)_{\Lambda_{\Sigma}}, \Lambda_{\Sigma}^{\infty} ; \mathbb{Z}\right)$. So

$$
\int_{\mathrm{SYZ}\left(\mathcal{L}_{\vec{l}}\right)} e^{-\frac{\widetilde{W}}{z}} \Omega=I_{A}\left(\mathcal{L}_{\vec{l}}\right)
$$

\section{Oscillatory integrals and Picard-Fuchs EQuations}

4.1. GKZ system. Following Iritani $\left[22\right.$, define the $H_{\mathrm{CR}, \widetilde{\mathbb{T}}}^{*}(\mathcal{X} ; \mathbb{C})$-valued equivariant $I$-function as below.

\section{Definition 4.1.}

$$
I^{\widetilde{\mathbb{T}}}\left(q_{0}, q, z\right)=e^{\left(t_{0}+\sum_{a=1}^{k} t_{a} p_{a}^{\widetilde{\mathbb{T}}}\right) / z} \sum_{d \in \mathbb{K}_{\text {eff }}} q^{d} \frac{\prod_{\{m\}=\left\{\left\langle D_{i}, d\right\rangle\right\}, m \leq 0}\left(\bar{D}_{i}^{\widetilde{\mathbb{T}}}+m z\right)}{\prod_{\{m\}=\left\{\left\langle D_{i}, d\right\rangle\right\}, m \leq\left\langle D_{i}, d\right\rangle}\left(\bar{D}_{i}^{\widetilde{\mathbb{T}}}+m z\right)} \mathbf{1}_{v(d)},
$$

where $p_{a}^{\widetilde{\mathbb{T}}}=\left(c_{1}\right)_{\widetilde{\mathbb{T}}}\left(\mathcal{L}_{e_{a}^{\vee}}\right), t_{a}=\log q_{a}$ for $a=0, \ldots, k$. 
The pullbacks of $I^{\widetilde{\mathbb{T}}}$ to torus fixed points in $\mathcal{I X}$ are functions such that the oscillatory integral $I_{\Xi}$ is a linear combination of them. Iritani has shown this in 22, with slightly different symbols and set of parameters. We quote his arguments and follow his notions, which lead to Proposition 4.2 in our notions. In the following text, $\mathrm{w}_{1}, \ldots, \mathrm{w}_{r}$ form a basis of $H_{\widetilde{\mathbb{T}}}^{*}$ (pt) (A-model), and let $w_{1}, \ldots, w_{r}, \lambda_{1}, \ldots, \lambda_{r}$ be complex numbers (B-model). Notice that $\mathrm{w}_{i}=-\left(c_{1}\right)_{\widetilde{\mathbb{T}}}\left(\mathcal{O}_{\mathbb{P} \infty}(-1)\right)$. Here we fix $H_{\widetilde{\mathbb{T}}}^{*}(\mathcal{I X})$-valued function $\left(\rho_{a}=\sum_{i=1}^{r} l_{i}^{(a)}\right)$

$$
H^{\widetilde{\mathbb{T}}}(q, z)=(-1)^{n} z^{-\frac{\omega_{1}+\cdots+\omega_{r}}{2 \pi \sqrt{-1}}} \sum_{d \in \mathbb{K}_{\text {eff }}} \frac{\prod_{a=1}^{k}\left(\frac{q_{a}}{z^{\rho a}}\right)^{\frac{p_{a}^{\widetilde{T}}}{2 \pi \sqrt{-1}}} q^{d} \mathbf{1}_{\operatorname{inv}(v(d))}}{z^{\left\langle D_{1}+\cdots+D_{r}, d\right\rangle} \prod_{i=1}^{r} \Gamma\left(1+\left\langle D_{i}, d\right\rangle+\frac{\bar{D}_{i}^{\widetilde{T}}}{2 \pi \sqrt{-1}}\right)} .
$$

Let $\iota_{\sigma, v}: \mathfrak{p}_{\sigma, v} \hookrightarrow \mathcal{I X}$ be a $\mathbb{T}$-fixed point. Then define

$$
H_{\sigma, v}(q, z)=\iota_{\sigma, v}^{*} H^{\widetilde{\mathbb{T}}}(q, z), \quad I_{\sigma, v}(q, z)=\iota_{\sigma, v}^{*} I^{\widetilde{\mathbb{T}}}\left(q_{0}, q, z\right) \in \widetilde{M} .
$$

Comparing with $\left.H_{\sigma, \operatorname{inv}(v)}\right|_{\mathrm{w}_{i}=2 \pi \sqrt{-1}} w_{i} / z$ with $\left.I_{\sigma, v}\right|_{\mathrm{w}_{i}=w_{i}}$ we find that

$$
\left.H_{\sigma, \operatorname{inv}(v)}\right|_{\mathrm{w}_{i}=2 \pi \sqrt{-1} w_{i} / z}=\left.c_{\sigma, v}\left(z, t_{0}, w_{i}\right) I_{\sigma, v}\right|_{\mathrm{w}_{i}=w_{i}}
$$

for $z>0$, where $t_{0} \in \mathbb{C}$ and generic $w_{i} \in \mathbb{C}$.

In [22, Lemma 4.19], because $H_{\sigma, v}$ are fundamental solutions to a GKZ system, the following integral can be expressed as a linear combination where $z>0, t_{0} \in \mathbb{C}$, generic $w_{i} \in \mathbb{C}$ and $0<q_{a}<\epsilon$ for some $\epsilon>0$

$$
\int_{\Xi} e^{-\frac{W}{z}-\frac{\lambda_{1}+\cdots+\lambda_{r}}{2 \pi \sqrt{-1}}} \Omega=\left.e^{\frac{-t_{0}}{z}} \sum_{(\sigma, v)} b_{\sigma, v}\left(\lambda_{i}\right) H_{\sigma, v}(q,-z)\right|_{\mathrm{w}_{i}=\lambda_{i}},
$$

Setting $\lambda_{i}=\frac{2 \pi \sqrt{-1} w_{i}}{z}$, we have the following proposition, where $h_{\sigma, v}\left(z, t_{0}, w_{i}\right)=$ $c_{\sigma, v}\left(-z, t_{0}, w_{i}\right) b_{\sigma, \operatorname{inv}(v)}$.

Proposition 4.2. For $z>0, t_{0} \in \mathbb{C}$, generic $w_{i} \in \mathbb{C}$ and $0<q_{a}<\epsilon$ with some $\epsilon>0$,

$$
I_{\Xi}=\left.\sum_{(\sigma, v)} h_{\sigma, v}\left(z, t_{0}, w_{i}\right) I_{\sigma, v}(q,-z)\right|_{\mathrm{w}_{i}=w_{i}} .
$$

4.2. Fixing the coefficients. For any $\mathbb{T}$-equivariant line bundle $\mathcal{L}$ on $\mathcal{X}$, define $\mathcal{L}_{v}=\left.\left(\operatorname{pr}^{*} \mathcal{L}\right)\right|_{\mathcal{X}_{v}}$. The stabilizer of $\mathcal{X}_{v}$ acts on the orbifold line bundle $\mathcal{L}_{v}$ on $\mathcal{X}_{v}$ by $\exp (2 \pi \sqrt{-1} f)$, where the rational number $0 \leq f<1$, called the age of $\mathcal{L}_{v}$ along $\mathcal{X}_{v}$. We denote the age of $\mathcal{L}_{v}$ along $\mathcal{X}_{v}$ by age ${ }_{v}(\mathcal{L})$. We define the following characteristic classes in $H_{\widetilde{\mathbb{T}}}^{*}(\mathcal{I X})(\mathcal{L}$ is also $\widetilde{\mathbb{T}}$-equivariant since the action of $\widetilde{\mathbb{T}}$ factors through $\mathbb{T})$

$$
\begin{aligned}
& \widetilde{c h}_{z}(\mathcal{L})=\bigoplus_{v \in \operatorname{Box}} e^{2 \pi \sqrt{-1}\left(-\frac{\left(c_{1}\right)_{\widetilde{T}}\left(\mathcal{L}_{v}\right)}{z}+\operatorname{age}_{v}(\mathcal{L})\right)}, \\
& \widetilde{\Gamma}_{z}(\mathcal{L})=\bigoplus_{v \in \operatorname{Box}} z^{\frac{\left(c_{1}\right) \widetilde{\pi}\left(\mathcal{L}_{v}\right)}{z}+1-\operatorname{age}_{v}(\mathcal{L})} \Gamma\left(\frac{\left(c_{1}\right)_{\widetilde{\mathbb{T}}}\left(\mathcal{L}_{v}\right)}{z}+1-\operatorname{age}_{v}(\mathcal{L})\right) .
\end{aligned}
$$

We understand these classes as series of cohomology classes. In particular we expand the Gamma function at $1-\operatorname{age}_{v}(\mathcal{L})>0$. We extend these classes to any $\mathcal{E} \in D^{b} \mathrm{Coh}_{\mathbb{T}}(\mathcal{X})$ via

$$
\begin{aligned}
\widetilde{c h}_{z}(\mathcal{E}) & =\widetilde{\mathrm{ch}}_{z}\left(\mathcal{E}_{1}\right)+\widetilde{\mathrm{ch}}_{z}\left(\mathcal{E}_{2}\right), \\
\widetilde{\Gamma}_{z}(\mathcal{E}) & =\widetilde{\Gamma}_{z}\left(\mathcal{E}_{1}\right) \widetilde{\Gamma}_{z}\left(\mathcal{E}_{2}\right),
\end{aligned}
$$

for any exact triangle $\mathcal{E}_{1} \rightarrow \mathcal{E} \rightarrow \mathcal{E}_{2} \rightarrow \mathcal{E}_{1}[1]$. 
Let $\sigma$ be a top-dimensional cone, and write $b_{j}=\sum_{b_{i} \in \sigma} s_{i j} b_{i}$. Then we have $D_{i}=-\sum_{b_{j} \notin \sigma} s_{i j} D_{j}$. The $\widetilde{\mathbb{T}}$-equivariant Chern roots of $\iota_{\sigma, v}^{*} T \mathcal{X}$ are $-\widetilde{\mathrm{w}}_{i}$ for $b_{i} \in \sigma$ where

$$
\widetilde{\mathrm{w}}_{i}=\mathrm{w}_{i}+\sum_{b_{j} \notin \sigma} s_{i j} \mathrm{w}_{j} .
$$

We also define complex numbers

$$
\widetilde{w}_{i}=w_{i}+\sum_{b_{j} \notin \sigma} s_{i j} w_{j} .
$$

Let $\mathcal{L}_{\vec{l}}$ be an ample line bundle, $\sigma$ be a top dimensional cone, and $v_{0} \in \sigma$. Define the piecewise Lagrangian for $t \geq 0$

$$
\Xi_{\sigma, t}\left(\mathcal{L}_{\vec{l}}\right)=\Xi\left(\mathcal{L}_{\vec{l}}\right)+t v_{0}=\sum_{\tau \in \Sigma}\left(-\tau+t v_{0}\right) \times \Delta_{\tau}(\vec{l}) .
$$

This Lagrangian is not conical for $t \neq 0$. They are all homotopic to each other, and corresponds to the same element in $H_{n}\left(\left(\overline{T^{*} M_{\mathbb{R}}}\right)_{\Lambda_{\Sigma}}, \Lambda_{\Sigma}^{\infty} ; \mathbb{Z}\right)$.

For a given top dimensional cone $\sigma$, there is a uniquely determined $\eta_{\sigma}$ such that $\eta_{\sigma}\left(\widetilde{b}_{i}\right)=0$, i.e. $\eta_{\sigma, i a}=0$ for $b_{i} \in \sigma$. By the fact that each $e_{a}^{\vee} \in \widetilde{\operatorname{Nef}}_{\sigma}$ and $e_{a}^{\vee}=\sum_{i=1}^{r} \eta_{\sigma, i a} D_{i}$, we know each $\eta_{\sigma, i a}>0$ for $b_{i} \notin \sigma$. So each $q_{\eta_{\sigma}, i}^{\prime}=\prod_{a=1}^{k} q_{a}^{\eta_{\sigma, i a}}$ for $b_{i} \notin \sigma$, and $\lim _{q \rightarrow 0} q_{\eta_{\sigma}}^{\prime}=0$. This limit is called the large radius limit when $\mathcal{X}$ is a smooth manifold.

For this $\sigma$ and $\eta_{\sigma}$, we define

$$
X_{i}^{\sigma}=X_{i}=Y^{b_{i}}, b_{i} \in \sigma ; \quad X_{i}^{\sigma}=Y^{b_{i}}, b_{i} \notin \sigma .
$$

The superpotential and the equivariantly perturbed superpotential are

$$
\begin{gathered}
W_{\eta}=\sum_{b_{i} \in \sigma} X_{i}+\sum_{b_{i} \notin \sigma} X_{i}=\sum_{b_{i} \in \sigma} X_{i}^{\sigma}+\sum_{b_{i} \notin \sigma} q_{\eta_{\sigma}, i}^{\prime} X_{i}^{\sigma}, \\
\widetilde{W}_{\eta}=W_{\eta}+\sum_{i=1}^{r} w_{i} x_{i} .
\end{gathered}
$$

We list a simple fact as a lemma, which we will use several times.

Lemma 4.3. For a top dimensional cone $\sigma$ and any given $A \gg 0$, we are able to choose $w_{1}, \ldots, w_{r}$ in an open region in $\mathbb{C}^{r}$ such that $\operatorname{Re} \widetilde{w}_{i}<-A, b_{i} \in \sigma$.

Proof. Let Re $w_{i} \ll 0$ for $b_{i} \in \sigma$ while keeping $w_{i}$ bounded for $b_{i} \notin \sigma$.

The map $\nu$ defined in Equation (5) identifies $\mathbb{K}_{\sigma} / \mathbb{L}$ with $\operatorname{Box}(\sigma)$. Since we have fixed an integral basis $e_{1}, \ldots, e_{k}$ of $\mathbb{L}$, this $\nu$ identifies $\left\{\sum_{i=1}^{k} t_{i} e_{i} \mid 0 \leq t_{i}<1\right\} \cap \mathbb{K}_{\sigma}$ with $\operatorname{Box}(\sigma)$. In the rest of this section we regard any $v \in \operatorname{Box}(\sigma)$ as an element in $\mathbb{K}_{\mathrm{eff}, \sigma}$.

Proposition 4.4. For any line bundle $\mathcal{L}_{\vec{l}}$, the coefficients in the decomposition of $I_{A}\left(\mathcal{L}_{\vec{l}}\right)$ in Proposition 4.2 are (notice that $h_{\sigma, v}$ do no depend on $t_{0}$ )

$$
h_{\sigma, v}\left(z, w_{i}\right)=\frac{\left.\iota_{\sigma, v}^{*}\left(\operatorname{inv}^{*}\left(\widetilde{\Gamma}_{z}(T \mathcal{X}) \widetilde{c h}_{z}\left(\mathcal{L}_{\vec{l}}\right)\right)\right)\right|_{\mathrm{w}_{i}=w_{i}}}{\left.\left|G_{\sigma}\right|\left(\iota_{\sigma, v}^{*} e_{\widetilde{\mathbb{T}}}(T \mathcal{I} \mathcal{X})\right)\right|_{\mathrm{w}_{i}=w_{i}}} .
$$

Proof. We just need to prove this statement for ample line bundle $\mathcal{L}_{\vec{l}}$. The proposition then follows from the additivity of $\widetilde{\mathrm{ch}}$ (Equation 13) and characteristic cycles (Proposition 3.7), together with the fact that $\mathbb{T}$-equivariant $\mathbb{Q}$-ample line bundles 
generate the equivariant $K$-group and the derived category $D^{b} \operatorname{Coh}_{\mathbb{T}}(\mathcal{X})$. Since the choice of $\eta$ does not affect the integral $I_{\Xi\left(\mathcal{L}_{\vec{l}}\right)}$, we simply need to consider

$$
\int_{\Xi\left(\mathcal{L}_{\vec{l}}\right)} e^{-\frac{\widetilde{W} \eta_{\sigma}}{z}} \Omega
$$

In this proof, the symbols $O()$ and $o()$ concern the limit $q \rightarrow 0$. We have

$$
\begin{aligned}
& \left|G_{\sigma}\right| e^{\frac{t_{0}}{z}} \prod_{j \notin \sigma}\left(q_{\eta_{\sigma}, j}^{\prime}\right)^{\frac{w_{j}}{z}} \int_{\Xi\left(\mathcal{L}_{\hat{l}}\right)} e^{-\frac{\widetilde{W}_{\eta}}{z}} \Omega=\int_{\Xi\left(\mathcal{L}_{\vec{l}}\right)} e^{-\frac{\Sigma_{b_{i} \epsilon \sigma} X_{i}}{z}-\frac{\Sigma_{b_{i} \xi \sigma} X_{i}}{z}} \prod_{i=1}^{r}\left(X_{i}^{\sigma}\right)^{-\frac{w_{i}}{z}} \prod_{b_{i} \in \sigma} \frac{d X_{i}^{\sigma}}{X_{i}^{\sigma}} \\
& =\int_{\Xi_{\sigma, t}\left(\mathcal{L}_{\hat{l}}\right)} e^{-\frac{\sum_{b_{i} \in \sigma} X_{i}}{z}-\frac{\sum_{b_{i} \notin \sigma} X_{i}}{z}} \prod_{i=1}^{r}\left(X_{i}^{\sigma}\right)^{-\frac{w_{i}}{z}} \prod_{b_{i} \in \sigma} \frac{d X_{i}^{\sigma}}{X_{i}^{\sigma}} .
\end{aligned}
$$

Suppose $N>0$, and let $f\left(q_{1}, \ldots, q_{a}\right)$ be a function of $q \in\left(\mathbb{R}_{>0}\right)^{r}$. We say $f(q)=O_{N}$ if $f\left(t q_{1}, \ldots, t q_{a}\right)=O\left(t^{N}\right)$ as $t \rightarrow 0$. Consider the expansion

$$
\begin{aligned}
& e^{-\frac{\sum_{b_{i} \notin \sigma} X_{i}^{\sigma}}{z}} \prod_{i=1}^{r}\left(X_{i}^{\sigma}\right)^{-\frac{w_{i}}{z}-1}=\sum_{\beta \in \mathbb{K}_{\mathrm{eff}, \sigma}} \prod_{b_{i} \notin \sigma} \frac{q^{\beta}}{\left\langle\beta, D_{i}\right\rangle !(-z)^{\left\langle\beta, D_{i}\right\rangle}} \prod_{b_{i} \in \sigma}\left(X_{i}^{\sigma}\right)^{-\left\langle\beta, D_{i}\right\rangle-\frac{\widetilde{w}_{i}}{z}-1} \\
= & \sum_{\beta \in \mathbb{K}_{\mathrm{eff}, \sigma},|\beta| \leq N} \prod_{b_{i} \notin \sigma} \frac{q^{\beta}}{\left\langle\beta, D_{i}\right\rangle !(-z)^{\left\langle\beta, D_{i}\right\rangle}} \prod_{b_{i} \in \sigma}\left(X_{i}^{\sigma}\right)^{-\left\langle\beta, D_{i}\right\rangle-\frac{\widetilde{w}_{i}}{z}-1}+f\left(q, X^{\sigma}, w_{i}\right) .
\end{aligned}
$$

Here the remaining terms $f\left(q, X^{\sigma}, w_{i}\right)$, and $|\beta| \leq N$ denotes the condition $\sum_{i=1}^{a}\left\langle\beta, e_{a}^{\vee}\right\rangle \leq$ $N$.

We first choose an $N \gg 0$, and by Lemma 4.3 we choose $w_{i}$ such that $\operatorname{Re}\left(-\frac{\widetilde{w}_{i}}{z}\right)>$ $N+1$. We have

$$
\begin{aligned}
& \int_{\Xi_{\sigma, t}\left(\mathcal{L}_{i}\right)} e^{-\frac{\sum_{b_{i} \epsilon \sigma} x_{i}}{z}-\frac{\sum_{b_{i} \notin \sigma} X_{i}}{z}} \prod_{i=1}^{r}\left(X_{i}^{\sigma}\right)^{-\frac{w_{i}}{z}} \prod_{b_{i} \in \sigma} \frac{d X_{i}^{\sigma}}{X_{i}^{\sigma}} \\
& =\int_{\Xi_{\sigma, t}\left(\mathcal{L}_{i}\right)} e^{-\frac{\sum_{b_{i} \in \sigma} X_{i}^{\sigma}}{z}}\left(\sum_{\beta \in \operatorname{Box}(\sigma)} \frac{q^{\beta} \prod_{b_{i} \in \sigma}\left(X_{i}^{\sigma}\right)^{-\left\langle\beta, D_{i}\right\rangle-\frac{\widetilde{w}_{i}}{z}-1}}{\prod_{b_{i} \notin \sigma}\left\langle\beta, D_{i}\right\rangle !(-z)^{\left\langle\beta, D_{i}\right\rangle}}\right) \prod_{b_{i} \in \sigma} d X_{i}^{\sigma} \\
& \quad+\int_{\Xi_{\sigma, t}\left(\mathcal{L}_{i}\right)} e^{-\frac{\Sigma_{b_{i} \in \sigma} X_{i}^{\sigma}}{z}} f\left(q, X^{\sigma}, w_{i}\right) \prod_{b_{i} \in \sigma} d X_{i}^{\sigma} \\
& =\sum_{\beta \in \mathbb{K}_{\mathrm{eff}, \sigma},|\beta| \leq N} \prod_{b_{i} \notin \sigma} \frac{q^{\beta}}{\left\langle\beta, D_{i}\right\rangle !(-z)^{\left\langle\beta, D_{i}\right\rangle}} \prod_{b_{i} \in \sigma} \frac{\Gamma\left(-\left\langle\beta, D_{i}\right\rangle-\frac{\widetilde{w}_{i}}{z}\right)}{z^{\left\langle\beta, D_{i}\right\rangle+\frac{\widetilde{w}_{i}}{z}}} e^{-2 \pi \sqrt{-1}\left(-\left\langle\beta, D_{i}\right\rangle-\frac{\widetilde{w}_{i}}{z}\right) l_{i}}+O_{N} .
\end{aligned}
$$

In the computation above, the first two expressions do not depend on the value of $t$. So taking $t \rightarrow \infty$, the first integral on the second expression becomes an integral on $N_{\mathbb{R}} \times \underline{\chi}_{\sigma}(\vec{l})$ - the Gamma function evaluates this integral. While the second integral on the second expression produces the $O_{N}$ term since $f\left(q, X^{\sigma}, w_{i}\right)=O_{N}$.

We compute the pull-back of the $I$-function to $\mathfrak{p}_{\sigma^{\prime}, v}$ where $v \in \operatorname{Box}\left(\sigma^{\prime}\right)$ for any top dimensional cone $\sigma^{\prime}$. Note that

$$
\iota_{\sigma, v}^{*} \bar{D}_{i}^{\widetilde{\mathbb{T}}}=-\widetilde{\mathrm{w}}_{i}, \quad b_{i} \in \sigma,
$$

while

$$
\iota_{\sigma^{\prime}, v}^{*}\left(\prod_{a=1}^{k} q_{a}^{\frac{p_{a}^{\widetilde{T}}}{z}}\right)=\prod_{b_{j} \notin \sigma^{\prime}} q_{\eta_{\sigma^{\prime}}, j}^{\prime \frac{w_{j}}{z}}, \quad \prod_{b_{i} \notin \sigma} q_{\eta_{\sigma}^{\prime}, i}^{\frac{w_{i}}{z}}=\prod_{b_{j} \notin \sigma \cup \sigma^{\prime}} q_{\eta_{\sigma^{\prime}}, j}^{\frac{w_{j}}{z}} \prod_{b_{j} \in\left(\sigma \backslash \sigma^{\prime}\right)} q_{\eta_{\sigma^{\prime}}, j}^{\prime \frac{\Sigma_{b_{i} \notin \sigma} s_{i j} w_{i}}{z}} .
$$


We compute:

$$
\begin{aligned}
& \left.I_{\sigma, v}(q,-z)\right|_{\mathfrak{w}_{i}=w_{i}} \\
= & e^{-\frac{t_{0}}{z}} \prod_{b_{j} \notin \sigma}\left(q_{\eta_{\sigma}, j}^{\prime}\right)^{-\frac{w_{j}}{z}}\left(\frac{q^{v}}{\prod_{b_{i} \notin \sigma}\left\langle v, D_{i}\right\rangle !(-z)^{\left\langle v, D_{i}\right\rangle}} \prod_{b_{i} \in \sigma} \frac{1}{(-z)^{\left\lceil\left\langle v, D_{i}\right\rangle\right\rceil}\left(\left\langle v, D_{i}\right\rangle+\frac{\widetilde{w}_{i}}{z}\right)_{\left\lceil\left\langle v, D_{i}\right\rangle\right\rceil}}+q^{v} O_{1}\right), \\
& \left.I_{\sigma^{\prime}, v}(q,-z)\right|_{\mathfrak{w}_{i}=w_{i}}=e^{-\frac{t_{0}}{z}} \prod_{b_{j} \notin \sigma^{\prime}}\left(q_{\eta_{\sigma^{\prime}}, j}^{\prime}\right)^{-\frac{w_{j}}{z}}\left(O\left(q^{v}\right)\right), \quad \sigma^{\prime} \neq \sigma .
\end{aligned}
$$

Here we adopt the Pochhammer symbol $(a)_{b}=\Gamma(a) / \Gamma(a-b+1)$. Then

$$
\begin{aligned}
& \left.\left|G_{\sigma}\right| e^{\frac{t_{0}}{z}} \prod_{b_{i} \notin \sigma}\left(q_{\eta_{\sigma}, i}^{\prime}\right)^{\frac{w_{i}}{z}} I_{\sigma, v}(q,-z)\right|_{\mathrm{w}_{i}=w_{i}} \\
= & \frac{q^{v}}{\prod_{b_{i} \notin \sigma}\left\langle v, D_{i}\right\rangle !(-z)^{\left\langle v, D_{i}\right\rangle}} \prod_{b_{i} \in \sigma} \frac{1}{(-z)^{\left\lceil\left\langle v, D_{i}\right\rangle\right\rceil}\left(\left\langle v, D_{i}\right\rangle+\frac{-\widetilde{w}_{i}}{-z}\right)_{\left\lceil v, D_{i}\right\rceil}}+q^{v} O_{1}, \\
& \left.\left|G_{\sigma}\right| e^{\frac{t_{0}}{z}} \prod_{b_{i} \notin \sigma}\left(q_{\eta_{\sigma}, i}^{\prime}\right)^{\frac{w_{i}}{z}} I_{\sigma^{\prime}, v}(q,-z)\right|_{\mathrm{w}_{i}=w_{i}}=\prod_{b_{j} \in\left(\sigma \backslash \sigma^{\prime}\right)} q_{\eta_{\sigma^{\prime}}, j}^{-\frac{w_{j}+\Sigma_{b_{i} \xi \sigma \sigma_{i j} w_{i}}^{z}}{z}} O(1) .
\end{aligned}
$$

We further choose $w_{1}, \ldots, w_{r}$ such that $\operatorname{Re} \widetilde{w}_{i}<-\max \{A, N\}$ for each $b_{i} \in \sigma$ by Lemma 4.3. Here $A=A(N)$ is sufficiently large such that for any $\sigma^{\prime} \neq \sigma$ and $b_{i} \in\left(\sigma \backslash \sigma^{\prime}\right), q_{\eta_{\sigma^{\prime}}, i}^{\prime-\frac{\widetilde{w}_{i}}{z}}=q^{\beta}$, and $|\beta|>N$. Comparing with the coefficients of $I_{\sigma, v}(q,-z)$, we find that

$$
\begin{aligned}
h_{\sigma, v}\left(z, w_{i}\right) & =\frac{1}{\left|G_{\sigma}\right|} \prod_{b_{i} \in \sigma} z^{-\frac{\widetilde{w}_{i}}{z}+\left\{-\left\langle v, D_{i}\right)\right\}} \Gamma\left(\frac{-\widetilde{w}_{i}}{z}+\left\{-\left\langle v, D_{i}\right\rangle\right\}\right) e^{-2 \pi \sqrt{-1}\left(-\left\langle v, D_{i}\right\rangle-\frac{\widetilde{w}_{i}}{z}\right) l_{i}} \\
& =\frac{\left.\iota_{\sigma, v}^{*}\left(\operatorname{inv}^{*}\left(\widetilde{\Gamma}_{z}(T \mathcal{X}) \widetilde{\mathrm{h}}_{z}\left(\mathcal{L}_{\vec{l}}\right)\right)\right)\right|_{\mathrm{w}_{i}=w_{i}}}{\left.\left|G_{\sigma}\right|\left(\iota_{\sigma, v}^{*} e_{\widetilde{\mathbb{T}}}(T \mathcal{I} \mathcal{X})\right)\right|_{\mathrm{w}_{i}=w_{i}}} .
\end{aligned}
$$

Here $\left(c_{1}\right)_{\widetilde{\mathbb{T}}}\left(\mathcal{L}_{\vec{l}}\right)=-\sum_{i \in \sigma} l_{i} \widetilde{\mathrm{w}}_{i}$, and $\operatorname{age}_{v}\left(\mathcal{L}_{\vec{l}}\right)=\sum_{b_{i} \in \sigma}\left\{-\left\langle v, D_{i}\right\rangle\right\} l_{i}$.

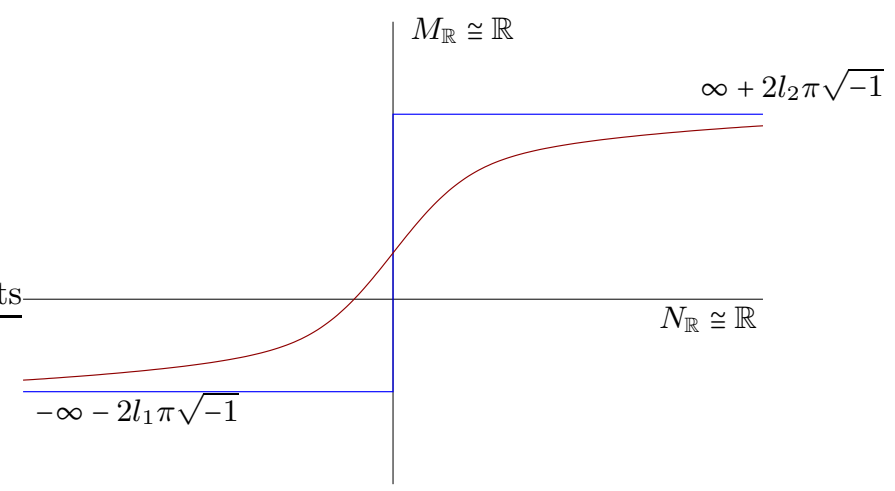

Figure 3 . The characteristic cycle (blue) $\operatorname{CC}\left(\mathcal{O}_{\mathbb{P}^{1}}\left(l_{1} \mathcal{D}_{1}+l_{2} \mathcal{D}_{2}\right)\right)$ and the SYZ dual of the same line bundle (red). They live in $T^{*} M_{\mathbb{R}} \cong N_{\mathbb{R}} \times M_{\mathbb{R}}$. 
Example 4.5 ([12]). $\mathcal{X}=\mathbb{P}^{1}, \sigma_{1}=\sigma=\mathbb{R}_{\geq 0}, \sigma_{2}=\mathbb{R}_{\leq 0}, \widetilde{W}_{\eta_{\sigma}}=X_{1}+\frac{q}{X_{1}}+w_{1} \log X_{1}+$ $w_{2} \log \frac{q}{X_{1}}$. Let $\mathcal{L}_{\vec{l}}=\mathcal{O}\left(l_{1} \mathcal{D}_{1}+l_{2} \mathcal{D}_{2}\right)$. When $q \rightarrow 0$,

$$
\begin{aligned}
e^{t_{0} / z} q^{w_{2} / z} \int_{\Xi\left(\mathcal{L}_{\vec{l}}\right)} e^{-\widetilde{W}_{\eta_{\sigma}} / z} \Omega & =\int_{\Xi\left(\mathcal{L}_{\vec{l}}\right)} e^{-\frac{X_{1}}{z}} e^{-\frac{q}{z X_{1}}} X_{1}^{\left(w_{1}-w_{2}\right) / z} d X_{1} / X_{1} \\
& \rightarrow z^{\frac{w_{2}-w_{1}}{z}} \Gamma\left(\frac{w_{2}-w_{1}}{z}\right) e^{\frac{2 \pi \sqrt{-1} l_{1}\left(w_{2}-w_{1}\right)}{z}}=\iota_{\sigma}^{*}\left(\widetilde{\Gamma}_{z}(T \mathcal{X}) \widetilde{\operatorname{ch}}\left(\mathcal{L}_{\vec{l}}\right)\right)_{\mathrm{w}_{i}=w_{i}} .
\end{aligned}
$$

Thus

Similarly

$$
h_{\sigma_{1}}=z^{\frac{w_{2}-w_{1}}{z}} \Gamma\left(\frac{w_{2}-w_{1}}{z}\right) e^{\frac{2 \pi \sqrt{-1} l_{1}\left(w_{2}-w_{1}\right)}{z}} .
$$

$$
h_{\sigma_{2}}=z^{\frac{w_{1}-w_{2}}{z}} \Gamma\left(\frac{w_{1}-w_{2}}{z}\right) e^{\frac{2 \pi \sqrt{-1} l_{2}\left(w_{1}-w_{2}\right)}{z}} .
$$

Comparing to the notion of [12], $\mathcal{D}_{1}=p_{2}$ and $\mathcal{D}_{2}=p_{1}$ for $p_{1}, p_{2}$ defined in [12].

Example 4.6. $\mathcal{X}$ is a semi-positive smooth projective toric variety.

$$
\begin{gathered}
\lim _{q \rightarrow 0} e^{t_{0} / z} \prod_{j \notin \sigma}\left(q_{\eta_{\sigma}, j}^{\prime}\right)^{\frac{w_{j}}{z}} \int_{\Xi\left(\mathcal{L}_{i}\right)} e^{-\frac{\widetilde{W}_{\eta_{\sigma}}}{z}} \Omega=\prod_{i \in \sigma} z^{-\frac{\widetilde{w}_{i}}{z}} \Gamma\left(-\frac{\widetilde{w}_{i}}{z}\right) e^{2 \pi \sqrt{-1} \frac{\widetilde{w}_{i}}{z} l_{i}}=\widetilde{\Gamma}_{z}(T \mathcal{X}) \widetilde{\operatorname{ch}}_{z}(\mathcal{L}), \\
\lim _{q \rightarrow 0} \iota_{\sigma}^{*}\left(e^{t_{0} / z} \prod_{j \notin \sigma} q_{\eta_{\sigma}, j}^{\frac{p_{\tilde{T}}}{z}} I^{\widetilde{\mathbb{T}}}\left(q_{0}, q,-z\right)\right)=1, \\
\int_{\Xi\left(\mathcal{L}_{i}\right)} e^{-\frac{\widetilde{W}}{z}} \Omega=\left.\sum_{\sigma} \iota_{\sigma}^{*}\left(\widetilde{\Gamma}_{z}(T X) \widetilde{c h}_{z}(\mathcal{L}) I^{\widetilde{\mathbb{T}}}\left(q_{0}, q,-z\right)\right)\right|_{w_{i}=w_{i}} .
\end{gathered}
$$

\section{Mirror theorem and Gromov-Witten potentials}

Definition 5.1. Let $\mathcal{X}$ be a complete toric orbifold. We define genus $g$, degree $d \in H_{2}(\mathcal{X} ; \mathbb{Z}), \widetilde{\mathbb{T}}$-equivariant descendant Gromov-Witten invariants of $\mathcal{X}$ as

$$
\left\langle\tau_{a_{1}}\left(\gamma_{1}\right) \ldots \tau_{a_{n}}\left(\gamma_{n}\right)\right\rangle_{g, n, d}^{\mathcal{X}}=\left\langle\gamma_{1} \psi_{1}^{a_{1}} \ldots \gamma_{n} \psi_{n}^{a_{n}}\right\rangle_{g, n, d}^{\mathcal{X}}=\int_{\left[\overline{\mathcal{M}}_{g, n}(\mathcal{X} ; d)\right]^{\mathrm{vir}}} \prod_{j=1}^{n} \psi_{j}^{a_{j}} \operatorname{ev}_{j}^{*}\left(\gamma_{j}\right) \in \mathbb{C}[\mathrm{w}],
$$

where $\gamma_{i} \in H_{\mathrm{CR}, \widetilde{\mathbb{T}}}^{*}(\mathcal{X})$ and $\mathrm{ev}_{j}: \overline{\mathcal{M}}(\mathcal{X} ; d) \rightarrow \mathcal{I X}$ is the $j$-th evaluation map. Let $\tau \in H_{\mathrm{CR}, \widetilde{T}}^{\leq 2}(\mathcal{X} ; \mathbb{C})$. We also define

$$
\begin{array}{r}
\left\langle\left\langle\tau_{a_{1}}\left(\gamma_{1}\right), \ldots, \tau_{a_{n}}\left(\gamma_{n}\right)\right\rangle_{g, n}^{\mathcal{X}}=\left\langle\left\langle\gamma_{1} \psi_{1}^{a_{1}}, \ldots, \gamma_{n} \psi_{n}^{a_{n}}\right\rangle_{g, n}^{\mathcal{X}}\right.\right. \\
=\sum_{d \geq 0} \sum_{\ell=0}^{\infty} \frac{1}{\ell !}\langle\tau_{a_{1}}\left(\gamma_{1}\right), \ldots, \tau_{a_{n}}\left(\gamma_{n}\right), \underbrace{\tau_{0}(\boldsymbol{\tau}), \ldots, \tau_{0}(\boldsymbol{\tau})}_{\ell \text { times }}\rangle_{g, n+\ell, d}^{\mathcal{X}} .
\end{array}
$$

We do not use Novikov variables since the convergence issue regarding $e^{\tau}$ is resolved in $[8,21$, or after invoking the mirror theorem and the oscillatory integral expression of the $I$-function. For the semi-positive $\mathcal{X}$, we quote the equivariant mirror theorem of toric stacks as below.

Theorem 5.2 (Coates-Corti-Iritani-Tseng 7, Cheong-Ciocan-Fontanine-Kim [6]). Let $\phi_{\alpha}$ be a basis of $H_{\mathrm{CR}, \widetilde{T}}^{*}(\mathcal{X} ; \mathbb{C})$ and $\phi^{\alpha}$ be its dual.

$$
\left\langle\frac{\phi_{\alpha}}{z(z-\psi)}\right\rangle_{0,1}^{\mathcal{X}} \phi^{\alpha}=I^{\widetilde{\mathbb{T}}}\left(q_{0}, q, z\right)
$$

under the mirror map

$$
\tau=\tau\left(q_{0}, q, z\right)
$$


Here we understand $\left\langle\frac{\phi_{\alpha}}{z(z-\psi)}\right\rangle_{0,1}^{\mathcal{X}}$ as a power series in $z^{-1}$. The mirror map is the coefficient of the $z^{-1}$-term in the expansion of $I^{\widetilde{\mathbb{T}}}$ :

$$
I^{\widetilde{\mathbb{T}}}\left(q_{0}, q, z\right)=\mathbf{1}+\frac{\boldsymbol{\tau}\left(q_{0}, q\right)}{z}+o\left(z^{-1}\right) .
$$

Define

$$
\phi_{\sigma, v}=\frac{\iota_{\sigma, v *} 1}{e_{\widetilde{\mathbb{T}}}\left(T_{\mathfrak{p}_{\sigma, v}} \mathcal{I X}\right)} .
$$

By the equivariant Atiyah-Bott localization, for any class $\alpha \in H_{\mathrm{CR}, \widetilde{\mathbb{T}}}^{*}(\mathcal{X} ; \mathbb{C})$,

$$
\alpha=\sum_{\sigma, v}\left(\iota_{\sigma, v}^{*} \alpha\right) \phi_{\sigma, v} .
$$

Let $\phi^{\sigma, v}$ be the dual basis to $\phi_{\sigma, v}$, and we have

$$
\phi^{\sigma, \operatorname{inv}(v)}=\left|G_{\sigma}\right| e_{\widetilde{\mathbb{T}}}\left(T_{\mathfrak{p}_{\sigma, v}} \mathcal{I} \mathcal{X}\right) \phi_{\sigma, v} .
$$

Theorem 5.3. For any $\mathbb{T}$-equivariant coherent sheaf $\mathcal{E}$ on $\mathcal{X}$, we define $\kappa(\mathcal{E})=$ $\widetilde{\Gamma}_{z}(T \mathcal{X}) \widetilde{c h}_{z}(\mathcal{E})$. Then for generic $w_{i} \in \mathbb{C}, z>0$ and $0<q_{a}<\epsilon$ where some small $\epsilon>0$

$$
I_{A}(\mathcal{E})=\int_{\Xi(\mathcal{E})} e^{-\frac{\widetilde{W}}{z}} \Omega=\left\langle\left.\left\langle\frac{\kappa(\mathcal{E})}{z(z+\psi)}\right\rangle_{0,1}^{\mathcal{X}}\right|_{w_{i}=w_{i}} .\right.
$$

Proof. We only need to prove this theorem when $\mathcal{E}=\mathcal{L}_{\vec{l}}$ since line bundles generates the equivariant K-group. Since

$$
I^{\widetilde{\mathbb{T}}}\left(q_{0}, q,-z\right)=\sum_{\sigma, v}\left(\iota_{\sigma, v}^{*} I^{\widetilde{\mathbb{T}}}\left(q_{0}, q,-z\right)\right) \phi_{\sigma, v},
$$

we know that

$$
\left.\left\langle\frac{\phi^{\sigma, v}}{z(z+\psi)}\right\rangle\right\rangle_{0,1}^{\mathcal{X}}=\iota_{\sigma, v}^{*} I^{\widetilde{\mathbb{T}}}\left(q_{0}, q,-z\right) .
$$

Then

$$
\begin{aligned}
I_{A}\left(\mathcal{L}_{\vec{l}}\right) & =\left.\sum_{\sigma, v} h_{\sigma, v}\left(z, w_{i}\right) \iota_{\sigma, v}^{*} I^{\widetilde{\mathbb{T}}}\left(q_{0}, q,-z\right)\right|_{\mathrm{w}_{i}=w_{i}} \\
& =\sum_{\sigma, v}\left\langle\left.\left\langle\frac{\left.\iota_{\sigma, v}^{*}\left(\operatorname{inv}^{*}\left(\widetilde{\Gamma}_{z}(T \mathcal{X}) \widetilde{c h}_{z}\left(\mathcal{L}_{\vec{l}}\right)\right)\right)\right|_{\mathrm{w}_{i}=w_{i}} \phi^{\sigma, v}}{\left|G_{\sigma}\right|\left(\iota_{\sigma, v}^{*}\left(e_{\widetilde{\mathbb{T}}}(T \mathcal{I} \mathcal{X})\right)\right)_{\mathrm{w}_{i}=w_{i}} z(z+\psi)}\right\rangle_{0,1}^{\mathcal{X}}\right|_{\mathrm{w}_{i}=w_{i}}\right. \\
& =\sum_{\sigma, v}\left\langle\left.\left\langle\frac{\left.\iota_{\sigma, v}^{*}\left(\operatorname{inv}^{*}\left(\widetilde{\Gamma}_{z}(T \mathcal{X}) \widetilde{c}_{z}\left(\mathcal{L}_{\vec{l}}\right)\right)\right)\right|_{\mathrm{w}_{i}=w_{i}} \phi_{\sigma, \operatorname{inv}(v)} e_{\widetilde{\mathbb{T}}}\left(T_{\mathfrak{p}_{\sigma, \operatorname{inv} v}} \mathcal{I} \mathcal{X}\right)}{\left(\iota_{\sigma, v}^{*}\left(e_{\widetilde{\mathbb{T}}}(T \mathcal{I} \mathcal{X})\right)\right)_{\mathrm{w}_{i}=w_{i}} z(z+\psi)}\right\rangle_{0,1}^{\mathcal{X}}\right|_{\mathrm{w}_{i}=w_{i}}\right. \\
& =\left\langle\left.\left\langle\frac{\widetilde{\Gamma}_{z}(T \mathcal{X}) \widetilde{c h}_{z}\left(\mathcal{L}_{\vec{l}}\right)}{z(z+\psi)}\right\rangle_{0,1}^{\mathcal{X}}\right|_{\mathrm{w}_{i}=w_{i}} .\right.
\end{aligned}
$$

Remark 5.4. By Remark 3.8, we have the following when $\mathcal{L}$ is an ample line bundle on smooth $\mathcal{X}$.

$$
\int_{\mathrm{SYZ}(\mathcal{L})} e^{-\widetilde{W} / z} \Omega=\left.\left\langle\frac{\kappa(\mathcal{L})}{z(z+\psi)}\right\rangle_{0,1}^{\mathcal{X}}\right|_{\mathrm{w}_{i}=w_{i}}
$$




\section{REFERENCES}

[1] M. Abouzaid, Morse homology, tropical geometry, and homological mirror symmetry for toric varieties, Selecta Math. (N.S.) 15 (2009), no. 2, 189-270.

[2] D. Arinkin and A. Polishchuk, Fukaya category and Fourier transform, Winter School on Mirror Symmetry, Vector Bundles and Lagrangian Submanifolds (Cambridge, MA, 1999), AMS/IP Stud. Adv. Math., vol. 23, Amer. Math. Soc., Providence, RI, 2001, pp. 261-274.

[3] L. A. Borisov, L. Chen, and G. G. Smith, The orbifold Chow ring of toric Deligne-Mumford stacks, J. Amer. Math. Soc. 18 (2005), no. 1, 193-215 (electronic).

[4] T. Bridgeland, Stability conditions on triangulated categories, Ann. of Math. (2) 166 (2007), no. $2,317-345$.

[5] W. Chen and Y. Ruan, A new cohomology theory of orbifold, Comm. Math. Phys. 248 (2004), no. $1,1-31$.

[6] D. Cheong, I. Ciocan-Fontanine, and B. Kim, Orbifold quasimap theory, Math. Ann. 363 (2015), no. 3-4, 777-816.

[7] T. Coates, A. Corti, H. Iritani, and H.-H. Tseng, A mirror theorem for toric stacks, Compos. Math. 151 (2015), no. 10, 1878-1912.

[8] T. Coates and H. Iritani, On the convergence of Gromov-Witten potentials and Givental's formula, Michigan Math. J. 64 (2015), no. 3, 587-631.

[9] B. Fang, C.-C. M. Liu, D. Treumann, and E. Zaslow, A categorification of Morelli's theorem, Invent. Math. 186 (2011), no. 1, 79-114.

[10] — T-duality and homological mirror symmetry for toric varieties, Adv. Math. 229 (2012), no. 3, 1875-1911.

[11] Math. Res. Not. IMRN 4 (2014), 914-954.

[12] B. Fang, C.-C. M. Liu, and Z. Zong, The Eynard-Orantin recursion and equivariant mirror symmetry for the projective line, Geom. Topol. (to appear). arXiv:1411.3557.

[13] K. Fukaya, Y.-G. Oh, H. Ohta, and K. Ono, Lagrangian Floer theory on compact toric manifolds. I, Duke Math. J. 151 (2010), no. 1, 23-174.

[14] L Lagrangian Floer theory on compact toric manifolds II: bulk deformations, Selecta Math. (N.S.) 17 (2011), no. 3, 609-711.

[15] S. Galkin, V. Golyshev, and H. Iritani, Gamma classes and quantum cohomology of Fano manifolds: gamma conjectures, Duke Math. J. 165 (2016), no. 11, 2005-2077.

[16] S. Galkin and H. Iritani, Gamma conjecture via mirror symmetry (2015). arXiv:1508.00719.

[17] A. Givental, Equivariant Gromov-Witten invariants, Internat. Math. Res. Notices 13 (1996), 613-663.

[18] - A mirror theorem for toric complete intersections, Topological field theory, primitive forms and related topics (Kyoto, 1996), Progr. Math., vol. 160, Birkhäuser Boston, Boston, MA, 1998, pp. 141-175.

[19] _ Elliptic Gromov-Witten invariants and the generalized mirror conjecture, Integrable systems and algebraic geometry (Kobe/Kyoto, 1997), World Sci. Publ., River Edge, NJ, 1998, pp. 107-155.

[20] S. Hosono, Central charges, symplectic forms, and hypergeometric series in local mirror symmetry, Mirror symmetry. V, AMS/IP Stud. Adv. Math., vol. 38, Amer. Math. Soc., Providence, RI, 2006, pp. 405-439.

[21] H. Iritani, Convergence of quantum cohomology by quantum Lefschetz, J. Reine Angew. Math. 610 (2007), 29-69.

[22] _ An integral structure in quantum cohomology and mirror symmetry for toric orbifolds, Adv. Math. 222 (2009), no. 3, 1016-1079.

[23] M. Kashiwara and P. Schapira, Sheaves on manifolds, Grundlehren der Mathematischen Wissenschaften [Fundamental Principles of Mathematical Sciences], vol. 292, Springer-Verlag, Berlin, 1994. With a chapter in French by Christian Houzel; Corrected reprint of the 1990 original.

[24] Y. Kawamata, Derived categories of toric varieties, Michigan Math. J. 54 (2006), no. 3, $517-535$.

[25] L. Katzarkov, M. Kontsevich, and T. Pantev, Hodge theoretic aspects of mirror symmetry, From Hodge theory to integrability and TQFT tt*-geometry, Proc. Sympos. Pure Math., vol. 78, Amer. Math. Soc., Providence, RI, 2008, pp. 87-174. 
[26] M. Kontsevich, Homological algebra of mirror symmetry, Proceedings of the International Congress of Mathematicians, Vol. 1, 2 (Zürich, 1994), Birkhäuser, Basel, 1995, pp. 120-139.

[27] (1998). http://www.math.uchicago.edu/ mitya/langlands/kontsevich.ps, Course at ENS.

[28] T. Kuwagaki, The nonequivariant coherent-constructible correspondence for toric surfaces (2015). arXiv:1507.05393.

[29] T. Kuwagaki, The nonequivariant coherent-constructible correspondence for toric stacks (2016). arXiv: 1610.03214.

[30] N. C. Leung, S.-T. Yau, and E. Zaslow, From special Lagrangian to Hermitian-Yang-Mills via Fourier-Mukai transform, Adv. Theor. Math. Phys. 4 (2000), no. 6, 1319-1341.

[31] B. Lian, K. Liu, and S.-T. Yau, Mirror principle I, Asian J. Math. 1 (1997), no. 4, 729-763.

[32] — Mirror principle II, Asian J. Math. 3 (1999), no. 1, 109-146.

[33] — Mirror principle III, Asian J. Math. 3 (1999), no. 4, 771-800.

[34] D. Nadler, Microlocal branes are constructible sheaves, Selecta Math. (N.S.) 15 (2009), no. 4, 563-619.

[35] D. Nadler and E. Zaslow, Constructible sheaves and the Fukaya category, J. Amer. Math. Soc. 22 (2009), no. 1, 233-286.

[36] S. Scherotzke and N. Sibilla, The non-equivariant coherent-constructible correspondence and a conjecture of King, Selecta Math. (N.S.) 22 (2016), no. 1, 389-416.

[37] A. Strominger, S.-T. Yau, and E. Zaslow, Mirror symmetry is T-duality, Nuclear Phys. B 479 (1996), no. 1-2, 243-259.

[38] W. Schmid and K. Vilonen, Characteristic cycles of constructible sheaves, Invent. Math. 124 (1996), no. 1-3, 451-502.

[39] H.-H. Tseng and D. Wang, Seidel Representations and quantum cohomology of toric stacks. arXiv: 1211.3204.

[40] D. Treumann, Remarks on the nonequivariant coherent-constructible correspondence for toric varieties (2010). arXiv:1006.5756.

[41] D. Vaintrob, The coherent-constructible correspondence and incomplete topologies. http://pentagrue.com/current.pdf.

[42] E. Zaslow, Topological orbifold models and quantum cohomology rings, Comm. Math. Phys. 156 (1993), no. 2, 301-331.

Bohan Fang, Beijing International Center for Mathematical Research, Peking UniVersity, 5 Yiheyuan Road, BeiJing 100871, China

E-mail address: bohanfang@gmail.com 